\title{
Target Characterization and Scattering Power Decomposition for Full and Compact Polarimetric SAR Data
}

\author{
Subhadip Dey, Graduate Student Member, IEEE, Avik Bhattacharya, Senior Member, IEEE, \\ Debanshu Ratha, Graduate Student Member, IEEE, Dipankar Mandal, Graduate Student Member, IEEE, and \\ Alejandro C. Frery, Senior Member, IEEE,
}

\begin{abstract}
In radar polarimetry, incoherent target decomposition techniques help extract scattering information from polarimetric SAR data. This is achieved either by fitting appropriate scattering models or by optimizing the received wave intensity through the diagonalization of the coherency (or covariance) matrix. As such, the received wave information depends on the received antenna configuration. Additionally, a polarimetric descriptor that is independent of the received antenna configuration might provide additional information which is missed by the individual elements of the coherency matrix. This implies that existing target characterization techniques might neglect this information. In this regard, we suitably utilize the $2 \mathrm{D}$ and 3D Barakat degree of polarization which is independent of the received antenna configuration to obtain distinct polarimetric information for target characterization. In this study, we introduce new roll-invariant scattering-type parameters for both full-polarimetric (FP) and compact-polarimetric (CP) SAR data. These new parameters jointly use the information of the $2 \mathrm{D}$ and 3D Barakat degree of polarization and the elements of the coherency (or covariance) matrix. We use these new scatteringtype parameters, which provide equivalent information as the Cloude $\alpha$ for FP SAR data and the ellipticity parameter $\chi$ for CP SAR data, to characterize various targets adequately. Additionally, we appropriately utilize these new scattering-type parameters to obtain unique non-model based three-component scattering power decomposition techniques. We obtain the evenbounce, and the odd-bounce scattering powers by modulating the total polarized power by a proper geometrical factor derived using the new scattering-type parameters for FP and CP SAR data. The diffused scattering power is obtained as the depolarized fraction of the total power. Moreover, due to the nature of its formulation, the decomposition scattering powers are nonnegative and roll-invariant while the total power is conserved. The proposed method is both qualitatively and quantitatively assessed utilizing the L-band ALOS-2 and C-band Radarsat-2 FP and the associated simulated CP SAR data.
\end{abstract}

Index Terms-Full polarimetry, Compact polarimetry, Target decomposition, scattering-type parameter, Target characterization

\section{INTRODUCTION}

$\mathbf{P}$ OLARIMETRIC decompositions aim, among other applications, to characterize scattering mechanisms from a tar-

S. Dey, A. Bhattacharya, D. Ratha, and D. Mandal are with the Microwave Remote Sensing Lab, Center of Studies in Resources Engineering, Indian Institute of Technology Bombay, India, e-mail: (sdey2307@gmail.com).

Alejandro C. Frery is with the Universidade Federal de Alagoas, Maceió, Brazil, and the Key Lab of Intelligent Perception and Image Understanding of the Ministry of Education, Xidian University, Xi'an, China. (e-mail: acfrery@laccan.ufal.br). get. Broadly categorizing, target decomposition techniques are divided into two distinct categories: coherent and incoherent. Coherent decomposition techniques utilize information from the $2 \times 2$ complex scattering matrix $\mathbf{S}$, whereas incoherent decompositions extract information in terms of the secondorder statistics from the $3 \times 3$ coherency $\mathbf{T}$ or the covariance C matrices.

Several methods have been developed to decompose the average covariance or coherency matrices. In this regard, either the eigenvalue/eigenvector based decomposition methods provide a unique solution to the scattering mechanisms [1], [2], or the roll-invariant scattering descriptors derived using the geodesic distance between Kennaugh matrices [3]. The interpretation of the scattering information is achieved by obtaining a set of unique roll-invariant parameters. On the other hand, model-based decomposition methods utilize the physical and geometrical properties of targets to extract scattering information from second-order statistics.

The pioneering work of Freeman and Durden [4] on the three-component scattering power decomposition (F3D) paved the way for model-based decomposition techniques. The scattering powers obtained from their method were based on the assumption of target reflection symmetry, i.e., $\left\langle S_{\mathrm{HH}} S_{\mathrm{HV}}^{*}\right\rangle=$ $\left\langle S_{\mathrm{VV}} S_{\mathrm{VH}}^{*}\right\rangle=0$. The Freeman and Durden decomposition model is simple and easy to implement and has been utilized for several applications as, for instance, unsupervised classification [5], [6].

However, the reflection symmetry assumption is seldom verified for most of the targets in a typical natural scenario. Therefore, the condition of uncorrelated co-polarized and cross-polarized components does not hold, i.e., $\left\langle S_{\mathrm{HH}} S_{\mathrm{HV}}^{*}\right\rangle \neq 0$, and $\left\langle S_{\mathrm{VV}} S_{\mathrm{HV}}^{*}\right\rangle \neq 0$. In such a condition, the cross-polarized component, $\left\langle\left|S_{\mathrm{HV}}\right|^{2}\right\rangle$, might be predominant.

In this regard, Yamaguchi et al. proposed a four-component model-based decomposition [7], which incorporates a helix as a fourth component.

In both the Freeman-Durden [4] and Yamaguchi [7] decompositions, the primary scatterer from vegetation canopy is modeled as a thin cylinder. However, such a description is often too simplistic for the complex structural configuration of most vegetation canopies.

Hence, Arii et al. [8] proposed an $n^{\text {th }}$ power of cosine squared function to describe such complex canopy structures. Nonetheless, this study considered the canopy scattering as the 
dominant mechanism. Similarly, Neumann et al. [9] proposed the retrieval of forest parameters using polarimetric interferometric SAR data. This study combined the physical modelbased decomposition with a random volume over the ground inversion approach.

van Zyl et al. [10] proposed constraints to grant nonnegative eigenvalues. Similarly, Cui et al. [11] performed the complete decomposition of the coherency matrix into one volume component and two single scattering components using a non-negative power constraint. Hence, this decomposition technique was able to overcome the negative power problem.

The aforementioned model-based decompositions did not take into account the target orientation aspect with the radar line of sight. Within a radar resolution cell, targets can be randomly oriented about the radar line of sight and, thus, can have diverse polarimetric responses. Several studies attempted to compensate this target orientation effect [12]-[15]. The central idea behind orientation compensation techniques is to reduce the contribution of the cross-polarized component. In other words, these techniques minimize the overestimation of the volume scattering power while increasing the even-bounce scattering power. Later, Bhattacharya et al. [16] utilized the degree of polarization of the scattered wave as an adaptive parameter to improve the scattering power components of the Yamaguchi four-component decomposition. Chen et al. [17] proposed a generalized double and odd-bounce scattering models by separating them with their independent orientation angles.

An alternative approach to determine the orientation of a target while improving the scattering powers is Statistical Information Theory. In this regard, Bhattacharya et al. [18] optimized the Hellinger distance between orthogonal and rotated urban targets to the radar line of sight to determine the orientation angle and, finally, modifying the Yamaguchi four-component decomposition powers. Later, Eltoft et al. [19] extended the model-based decomposition techniques by introducing higher-order distribution functions and radar texture models. An et al. [20] reconsidered the problem of negative scattering powers and the overestimation of the volume scattering component in the Freeman-Durden decomposition. They proposed a methodology to completely decompose an arbitrary coherency matrix into several polarimetric symmetry components.

Stability of decomposition powers poses a significant challenge in several model-based decompositions. Jiao et al. [21] proposed a stable three-component decomposition by solving a constraint optimization problem. Shuang et al. [22] combined a new condition with the Freeman-Durden decomposition to distinguish human-made structure and nature media after orientation angle compensation.

Chen et al. [23] provide a review of decomposition techniques using polarimetric SAR data.

Full polarimetric (FP) SAR data provides optimum performance in target characterization due to its complete radar target information content. However, compact polarimetric SAR data offers more information than a single or dual-polarized SAR data, while covering larger swath widths compared to FP SAR systems.
In Compact Polarimetric (CP) radars, the relative phase between the two received polarizations is retained, unlike the conventional dual-polarized SAR systems. In the $\pi / 4$ mode [24], the transmitted polarization is a superposition of the linear horizontal $(\mathrm{H})$ and vertical polarization $(\mathrm{V})$ oriented at $45^{\circ}$ to the horizontal. The dual-circular compact polarimetry (DCP) model proposed in [25] used right circular polarization on transmit and right and left circular polarization on receive. Furthermore, Raney [26] proposed a new hybrid-polarity architecture, consisting of circular transmit and orthogonal linear polarizations receive. This new hybrid-pol architecture preserves all the information of the DCP mode since the Stokes parameter of electromagnetic (EM) wave does not depend on the received polarization basis [27].

Raney et al. proposed the $m-\delta$ [26] and $m-\chi$ [28] decomposition methods for the hybrid-compact polarimetric SAR data, where $m$ indicates the degree of polarization of the scattered EM wave. The performance of the $m-\delta$ decomposition largely depends on the purity in the transmission polarization of the EM wave. Hence, the phase difference parameter, $\delta$, provides better results only when the transmitted wave is perfectly circular. On the contrary, the ellipticity, $\chi$, is robust towards the transmitting wave polarization. While characterizing the scattering phenomenon from the lunar surface, Raney et al. [28] pointed out the ability of the $m-\chi$ decomposition to resolve certain even-odd bounce scattering ambiguity over lunar crater walls. Later, the authors hypothesized that a threecomponent $(m-\chi-\psi)$ decomposition would be more appropriate to discriminate different scattering mechanisms with $a$ priori information of the transmitting ellipticity $(\chi)$ of the EM wave. This striking idea was exploited by Bhattacharya et al. [29] while proposing the $S-\Omega$ decomposition, where $\Omega$ depends on $m$, the transmitting wave ellipticity $\left(\chi_{t}\right)$ and orientation $\left(\psi_{t}\right)$ and the received wave ellipticity $\left(\chi_{r}\right)$ and orientation $\left(\psi_{r}\right)$.

Incoherent target decomposition techniques might not utilize complete polarimetric information present in SAR data. In particular, such techniques for full polarimetric SAR data optimize the received wave intensity through the diagonalization of the coherency (or covariance) matrix [2], [30], [31]. Hence, the information provided by a parameter which is received antenna basis invariant might be useful. In this respect, the degree of polarization obtained from the $n$ dimensional $(n D)$ coherency matrix proposed by Barakat [32], [33] can be suitably utilized to gain enhanced polarimetric information. The Barakat degree of polarization is linked to the polarimetric contribution of the Shannon entropy [34].

In this study, we jointly use the 3D and 2D Barakat degree of polarization [33], and the elements of the coherency (or covariance) matrix to obtain roll-invariant scattering-type parameters for both FP and CP monostatic SAR data. These parameters are equivalent to the Cloude and Pottier parameter $\alpha$ [1] for FP SAR data, and the wave ellipticity parameter $\chi$ for CP SAR data. Additionally, we propose novel threecomponent scattering power decomposition techniques for both FP and CP SAR data by utilizing the scattering-type parameter and the 3D and 2D Barakat degree of polarization. Unlike traditional model-based decompositions, the pro- 
posed methods do not utilize any particular scattering models for the estimation of the scattering powers, and the formulations of these two techniques are equivalent for both full and compact polarimetric SAR data. Moreover, each power component is guaranteed to be non-negative, and the total power is conserved. The proposed "non-model" based three-component scattering power decomposition techniques are applied to full and simulated hybrid-compact polarimetric L-band ALOS-2 and C-band RS-2 SAR data over Mumbai and San Francisco, respectively. The effectiveness of the proposed methods is used for qualitative analysis of scattering mechanisms and quantitative analysis of the scattering powers.

This work unfolds as follows. We derive the new target scattering-type parameters for full and compact polarimetric SAR data in Section II; those parameters are further utilized to obtain unique three-component non-model based scattering power decompositions for two datasets. In Section III, we compare the results obtained from the proposed techniques with other existing target characterization parameters and decomposition techniques. Sections III-A1 and III-B1 compare the proposed target characterization parameters with the ones existing in literature for FP and CP data. Accordingly, sections III-A2 and III-B2 present and compare the proposed 3component non-model based decomposed powers with two decomposition techniques for FP and CP SAR data, respectively. Finally, Section IV summarizes the proposed methodologies and concludes by highlighting its advantages and limitations for different SAR data.

\section{Methodology}

We introduce new roll-invariant scattering-type parameters by utilizing the 3D and 2D Barakat degree of polarization [33] ( $m$ ) and the elements of the $3 \times 3$ coherency and the $2 \times 2$ covariance matrix for both FP and CP SAR data, respectively. In this regard, we use the expression derived from [33] to calculate the 3D and 2D Barakat degree of polarization. Subsequently, we use these scattering-type parameters to obtain non-model based three-component scattering power decompositions for both FP and CP SAR data. We obtain the evenbounce and the odd-bounce powers by modulating the total polarized power by a specific geometrical factor easily derived using the new scattering-type parameters for both FP and CP SAR data.

\section{A. Full Polarimetry}

In FP SAR, the $2 \times 2$ complex scattering matrix $\mathbf{S}$ encompasses complete polarimetric information about backscattering from targets for each pixel. It is expressed in the backscatter alignment (BSA) convention in the linear horizontal $(\mathrm{H})$ and linear vertical $(\mathrm{V})$ polarization basis as,

$$
\mathbf{S}=\left[\begin{array}{ll}
S_{\mathrm{HH}} & S_{\mathrm{HV}} \\
S_{\mathrm{VH}} & S_{\mathrm{VV}}
\end{array}\right] \Rightarrow \boldsymbol{k}=V([\mathbf{S}])=\frac{1}{2} \operatorname{Tr}(\mathbf{S} \Psi)
$$

where $V(\cdot)$ is the vectorization operator on the scattering matrix, $\Psi$ is the corresponding basis matrix, and $\operatorname{Tr}$ is the sum of the diagonal elements of the matrix. Each element of the matrix represents the backscattering response of the target at a specific polarization. The diagonal elements of the matrix represent the co-polarized scattering information, while the off-diagonal terms represent the cross-polarized information. In the monostatic backscattering case, the reciprocity theorem constrains the scattering matrix to be symmetric, i.e., $S_{\mathrm{HV}}=S_{\mathrm{VH}}$.

The multi-looked Hermitian positive semi-definite $3 \times 3$ coherency matrix $\mathbf{T}$ is obtained from the averaged outer product of the target vector $\boldsymbol{k}_{P}$ (derived using the Pauli basis matrix, $\Psi_{P}$ ) with its conjugate. Similarly, the $3 \times 3$ covariance matrix $\mathbf{C}$ is obtained from the averaged outer product of the target vector $\boldsymbol{k}_{L}$ (derived using the Lexicographic basis matrix, $\Psi_{L}$ ) with its conjugate.

$$
\begin{aligned}
& \Psi_{P}=\left\{\sqrt{2}\left[\begin{array}{ll}
1 & 0 \\
0 & 1
\end{array}\right], \sqrt{2}\left[\begin{array}{cc}
1 & 0 \\
0 & -1
\end{array}\right], \sqrt{2}\left[\begin{array}{ll}
0 & 1 \\
1 & 0
\end{array}\right]\right\}, \\
& \Psi_{L}=\left\{2\left[\begin{array}{ll}
1 & 0 \\
0 & 0
\end{array}\right], 2 \sqrt{2}\left[\begin{array}{ll}
0 & 1 \\
0 & 0
\end{array}\right], 2\left[\begin{array}{ll}
0 & 0 \\
0 & 1
\end{array}\right]\right\} .
\end{aligned}
$$

Similar to the conventional degree of polarization, the 3D Barakat degree of polarization $(0 \leq m \leq 1)$ also characterizes the state of polarization (or purity) of an EM wave. For a completely polarized EM wave, $m=1$ and for a completely unpolarized EM wave, $m=0$. In between these two extreme cases, the EM wave is said to be partially polarized, $0<m<$ 1.

Barakat [32] provided an expression of $m$ for the $n \times n$ coherency matrix. This expression is used in this study to obtain the 3D Barakat degree of polarization $m_{\mathrm{FP}}$ from the $3 \times 3$ coherency matrix $\mathbf{T}$ for FP SAR data as,

$$
m_{\mathrm{FP}}=\sqrt{1-\frac{27|\mathbf{T}|}{(\operatorname{Tr}(\mathbf{T}))^{3}}},
$$

where $|\cdot|$ is the determinant of a matrix. It should be noted that although this quantity is related to the conventional degree of polarization, it is not the overall degree of polarization for the full-polarimetric case as it does not include all the invariants. The coherency (or covariance) matrix can be used to estimate this quantity considering suitable ergodicity properties.

Let us assume that

$$
\tan \eta_{1}=\frac{T_{11}}{m_{\mathrm{FP}} \operatorname{Span}} \quad \text { and } \tan \eta_{2}=\frac{T_{22}+T_{33}}{m_{\mathrm{FP}} \operatorname{Span}},
$$

where $T_{11}, T_{22}$, and $T_{33}$ are the diagonal elements of the coherency matrix, and denote

$$
\text { Span }=T_{11}+T_{22}+T_{33}
$$

Therefore, by using a simple relationship, we obtain:

$$
\begin{aligned}
\tan \theta_{\mathrm{FP}} & =\tan \left(\eta_{1}-\eta_{2}\right) \\
& =\frac{m_{\mathrm{FP}} \operatorname{Span}\left(T_{11}-T_{22}-T_{33}\right)}{T_{11}\left(T_{22}+T_{33}\right)+m_{\mathrm{FP}}^{2} \operatorname{Span}^{2}} .
\end{aligned}
$$

Appendices $\mathrm{A} 1$ and $\mathrm{B}$ prove that $\theta_{\mathrm{FP}} \in\left[-45^{\circ}, 45^{\circ}\right]$ is a rollinvariant parameter. This parameter can be used to characterize scattering-type information from targets.

It can be observed from equation (5), that when $m_{\mathrm{FP}}=0$ (i.e. when no polarization structure exists in the scattered EM wave), then $\theta_{\mathrm{FP}}=0$. Whereas, when $m_{\mathrm{FP}}=1$, then either 
$\theta_{\mathrm{FP}}=-45^{\circ}$ or $\theta_{\mathrm{FP}}=45^{\circ}$, depending on the scattering from a dihedral or a trihedral target respectively. Otherwise, for all other cases, $\theta_{\mathrm{FP}} \in\left(-45^{\circ}, 45^{\circ}\right)$.

We split the total power (Span) into two components: evenbounce $\left(P_{d}^{\mathrm{FP}}\right)$ and odd-bounce $\left(P_{s}^{\mathrm{FP}}\right)$ scattering powers using a geometrical factor $\left(1 \pm \sin 2 \theta_{\mathrm{FP}}\right)$ using the $3 \mathrm{D}$ Barakat degree of polarization $m_{\mathrm{FP}}(2)$ and the scattering-type information $\theta_{\mathrm{FP}}(5)$. The diffused scattering power $\left(P_{v}^{\mathrm{FP}}\right)$ is obtained as the depolarized fraction of the total power:

$$
\begin{aligned}
& P_{d}^{\mathrm{FP}}=\frac{m_{\mathrm{FP}} \operatorname{Span}}{2}\left(1-\sin 2 \theta_{\mathrm{FP}}\right), \\
& P_{v}^{\mathrm{FP}}=\operatorname{Span}\left(1-m_{\mathrm{FP}}\right), \text { and } \\
& P_{s}^{\mathrm{FP}}=\frac{m_{\mathrm{FP}} \operatorname{Span}}{2}\left(1+\sin 2 \theta_{\mathrm{FP}}\right) .
\end{aligned}
$$

When $m_{\mathrm{FP}}=0$, then $P_{d}^{\mathrm{FP}}=P_{s}^{\mathrm{FP}}=0$, and $P_{v}^{\mathrm{FP}}=$ Span. This corresponds to the complete depolarized case. For pure even-bounce scattering, $m_{\mathrm{FP}}=1$ and $\theta_{\mathrm{FP}}=-45^{\circ}$ with $P_{s}^{\mathrm{FP}}=$ $P_{v}^{\mathrm{FP}}=0$, and $P_{d}^{\mathrm{FP}}=$ Span. For pure odd-bounce scattering, $m_{\mathrm{FP}}=1$ and $\theta_{\mathrm{FP}}=45^{\circ}$ with $P_{d}^{\mathrm{FP}}=P_{v}^{\mathrm{FP}}=0$, and $P_{s}^{\mathrm{FP}}=$ Span. It is noteworthy that the scattering power components are non-negative, and that the total power (Span) is conserved for any polarization state.

\section{B. Compact Polarimetry}

The hybrid compact polarimetric mode measures a projection of the $2 \times 2$ complex scattering matrix $\mathbf{S}$ as,

$$
\begin{aligned}
{\left[\begin{array}{l}
E_{C \mathrm{H}} \\
E_{C \mathrm{~V}}
\end{array}\right] } & =\frac{1}{\sqrt{2}}\left[\begin{array}{ll}
S_{\mathrm{HH}} & S_{\mathrm{HV}} \\
S_{\mathrm{VH}} & S_{\mathrm{VV}}
\end{array}\right]\left[\begin{array}{c}
1 \\
\pm i
\end{array}\right] \\
& =\frac{1}{\sqrt{2}}\left[\begin{array}{l}
S_{\mathrm{HH}} \pm i S_{\mathrm{HV}} \\
S_{\mathrm{VH}} \pm i S_{\mathrm{VV}}
\end{array}\right]
\end{aligned}
$$

where the subscript $C$ can be either the left-hand circular (LHC) transmit with a + sign or the right-hand circular (RHC) transmit with a - sign. The $2 \times 2$ covariance matrix $\mathbf{C}_{2}$ is then obtained from the elements of the scattering vector as

$$
\mathbf{C}_{2}=\left[\begin{array}{cc}
\left\langle\left|E_{C \mathrm{H}}\right|^{2}\right\rangle & \left\langle E_{C \mathrm{H}} E_{C \mathrm{~V}}^{*}\right\rangle \\
\left\langle E_{C \mathrm{~V}} E_{C \mathrm{H}}^{*}\right\rangle & \left\langle\left|E_{C \mathrm{~V}}\right|^{2}\right\rangle
\end{array}\right] .
$$

For CP-SAR data, the $4 \times 1$ Stokes vector $\overrightarrow{\mathbf{S}}$ can be written in terms of the elements of the $2 \times 2$ covariance matrix $\mathbf{C}_{2}$ :

$$
\overrightarrow{\mathbf{S}}=\left[\begin{array}{c}
S_{0} \\
S_{1} \\
S_{2} \\
S_{3}
\end{array}\right]=\left[\begin{array}{c}
C_{11}+C_{22} \\
C_{11}-C_{22} \\
C_{12}+C_{21} \\
\pm j\left(C_{12}-C_{21}\right)
\end{array}\right]
$$

where \pm corresponds to the left and right circular polarizations, respectively.

The first element of the Stokes vector, $S_{0}$, is a measure of the total average received power. The third element, $S_{3}$, is a measure of the average received power in circular polarization. The handedness of this circular polarization can be inferred from the sign $( \pm)$ of the $S_{3}$ component.

The proportion of the power that is received by the radar in opposite-sense circular polarization to that transmitted is $\left(S_{0}+S_{3}\right) / 2$. For example, OC $=\left(S_{0}+S_{3}\right) / 2=\left|E_{\mathrm{R}}\right|^{2}$ for left-circular (L) polarization on transmit, where $\left|E_{\mathrm{R}}\right|^{2}$ is the intensity of the right-circular component. Such a change of polarity occurs when an EM wave undergoes an odd number of reflections.

Similarly, $\left(S_{0}-S_{3}\right) / 2$ is a measure of the power received by the radar in the same-sense circular polarization as it was transmitted, which represents an even number of reflections. For example, $\mathrm{SC}=\left(S_{0}-S_{3}\right) / 2=\left|E_{\mathrm{L}}\right|^{2}$ for left-circular (L) polarization on transmit, where $\left|E_{\mathrm{L}}\right|^{2}$ is the intensity of the left-circular component.

Similarly to the FP case, we use the Barakat formulation to obtain the 2D Barakat degree of polarization $m_{\mathrm{CP}}$ from the $2 \times 2$ covariance matrix $\mathbf{C}_{2}$ for CP SAR data as,

$$
m_{\mathrm{CP}}=\sqrt{1-\frac{4\left|\mathbf{C}_{2}\right|}{\left(\operatorname{Tr}\left(\mathbf{C}_{2}\right)\right)^{2}}} .
$$

Similarly to the FP case, let us assume

$$
\tan \zeta_{1}=\frac{\mathrm{OC}}{m_{\mathrm{CP}} S_{0}} \quad \text { and } \quad \tan \zeta_{2}=\frac{\mathrm{SC}}{m_{\mathrm{CP}} S_{0}},
$$

with,

$$
S_{0}=\mathrm{SC}+\mathrm{OC}
$$

Therefore, by using a simple relationship, we obtain,

$$
\begin{aligned}
\tan \theta_{\mathrm{CP}} & =\tan \left(\zeta_{1}-\zeta_{2}\right) \\
& =\frac{m_{\mathrm{CP}} S_{0}(\mathrm{OC}-\mathrm{SC})}{\mathrm{OC} \times \mathrm{SC}+m_{\mathrm{CP}}^{2} S_{0}^{2}}
\end{aligned}
$$

It can be observed from appendix A2 that $\theta_{\mathrm{CP}} \in\left[-45^{\circ}, 45^{\circ}\right]$ characterizes scattering-type information from targets utilizing the same sense circular (SC) and opposite sense circular (OC) polarization scattered powers.

From (15), when $m_{\mathrm{CP}}=0$, i.e., when no polarization structure exists in the EM wave, then $\theta_{\mathrm{CP}}=0$. Whereas, when $m_{\mathrm{CP}}=1$, then either $\theta_{\mathrm{CP}}=-45^{\circ}$ or $\theta_{\mathrm{CP}}=45^{\circ}$, depending on the sense (i.e., right or left circular) of the received polarization with respect to the transmit polarization. Otherwise, for all other cases, $\theta_{\mathrm{CP}} \in\left(-45^{\circ}, 45^{\circ}\right)$.

Analogously to the FP case, the 2D Barakat degree of polarization $m_{\mathrm{CP}}$, and the scattering-type information $\theta_{\mathrm{CP}}$, can be used to split the total average received power $\left(S_{0}\right)$ into two components: even-bounce $\left(P_{d}^{\mathrm{CP}}\right)$, and odd-bounce $\left(P_{s}^{\mathrm{CP}}\right)$ using a geometrical factor $\left(1 \pm \sin 2 \theta_{\mathrm{CP}}\right)$. Similarly, the diffused scattering power $\left(P_{v}^{\mathrm{CP}}\right)$ is the depolarized fraction of the total power:

$$
\begin{aligned}
P_{d}^{\mathrm{CP}} & =\frac{m_{\mathrm{CP}} S_{0}}{2}\left(1-\sin 2 \theta_{\mathrm{CP}}\right), \text { and } \\
P_{v}^{\mathrm{CP}} & =S_{0}\left(1-m_{\mathrm{CP}}\right), \\
P_{s}^{\mathrm{CP}} & =\frac{m_{\mathrm{CP}} S_{0}}{2}\left(1+\sin 2 \theta_{\mathrm{CP}}\right)
\end{aligned}
$$

In the case of scattering power decomposition, when $m_{\mathrm{CP}}=$ $0, P_{d}^{\mathrm{CP}}=P_{s}^{\mathrm{CP}}=0$, and $P_{v}^{\mathrm{CP}}=S_{0}$. This corresponds to the complete depolarized case. For pure even-bounce scattering, $m_{\mathrm{CP}}=1$ and $\theta_{\mathrm{CP}}=-45^{\circ}$ with $P_{s}^{\mathrm{CP}}=P_{v}^{\mathrm{CP}}=0$, and $P_{d}^{\mathrm{CP}}=$ $S_{0}$. For pure odd-bounce scattering, $m_{\mathrm{CP}}=1$ and $\theta_{\mathrm{CP}}=45^{\circ}$ with $P_{d}^{\mathrm{CP}}=P_{v}^{\mathrm{CP}}=0$, and $P_{s}^{\mathrm{CP}}=S_{0}$. Moreover, the total power $\left(S_{0}\right)$ is conserved for any polarization state]. 


\section{RESULTS}

In the previous section, we derived two roll-invariant scattering-type parameters, $\theta_{\mathrm{FP}}$ and $\theta_{\mathrm{CP}}$ by jointly utilizing the $3 \mathrm{D}$ and 2D Barakat degree of polarization and the elements of the coherency and covariance matrices for FP and CP imaging modes respectively. Nevertheless, their physical interpretation is comparable to some of the established parameters known in the literature, viz., Cloude and Pottier's $\alpha$ [35] for FP SAR data, and Raney et al.'s $\chi$ [28] for CP SAR data. This section provides a comparison of $\theta_{\mathrm{FP}}$ and $\theta_{\mathrm{CP}}$ with $\bar{\alpha}=45^{\circ}-\alpha$ and $\bar{\chi}=-\chi$ respectively. The translations of $\alpha$ and $\chi$ to $\bar{\alpha}$ and $\bar{\chi}$, respectively, are presented solely to compare the scattering nature of the targets in the same range. We have also briefly analyzed the polarimetric scattering entropy, $H$ for the two imaging modes over some regions of interest.

We used two full polarimetric (FP) SAR images over Mumbai, India, and San Francisco (SF), USA, shown in Fig. 1. The Mumbai scene is an L-band ALOS-2 image with a center incidence angle of $33^{\circ}$. The image is multilooked by a factor of 3 in range direction, and 5 in the azimuth direction to generate $15 \mathrm{~m}^{2}$ pixels.

The SF scene is a C-band RS-2 image acquired with near and far range incidence angles of $28.02^{\circ}$ and $29.81^{\circ}$, respectively. The single look complex (SLC) image is multilooked by a factor of 2 in the range direction, and 4 in the azimuth direction to generate a $20 \mathrm{~m}^{2}$ ground pixel.

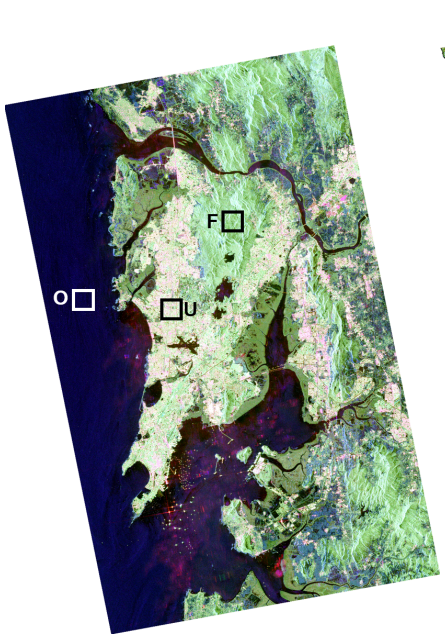

(a) Pauli RGB, Mumbai

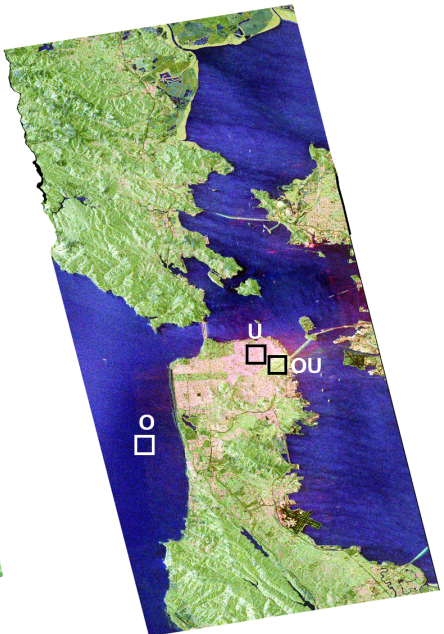

(b) Pauli RGB, San Francisco
Fig. 1. Pauli RGB images of ALOS-2 L-band (left) acquisition over Mumbai, India and RS-2 C-band (right) acquisition over San Francisco, USA.

Additionally, we generated simulated hybrid-compact polarimetric (CP) data from both the FP SAR data sets with an ellipticity angle of $-45^{\circ}$ (right circular) and $0^{\circ}$ orientation angle.

\section{A. Full polarimetry}

Figures 2 and 3 show the images of $\theta_{\mathrm{FP}}$ and $\bar{\alpha}$ for the ALOS2 L-band and RS-2 C-band SAR data, respectively. It can be seen that $\theta_{\mathrm{FP}}$ provides better contrast over different land cover classes with two different frequencies than $\bar{\alpha}$.

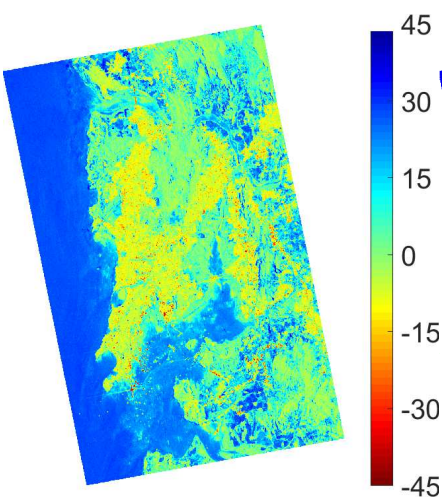

(a) $\bar{\alpha}$

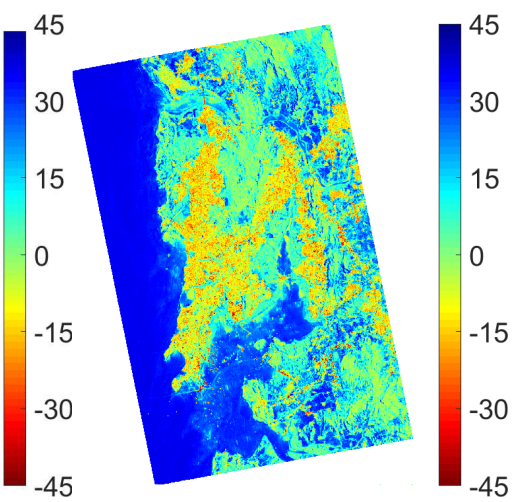

(b) $\theta_{\mathrm{FP}}$
Fig. 2. Images of $\bar{\alpha}$ and $\theta_{\mathrm{FP}}$ for ALOS-2 FP SAR data over Mumbai.

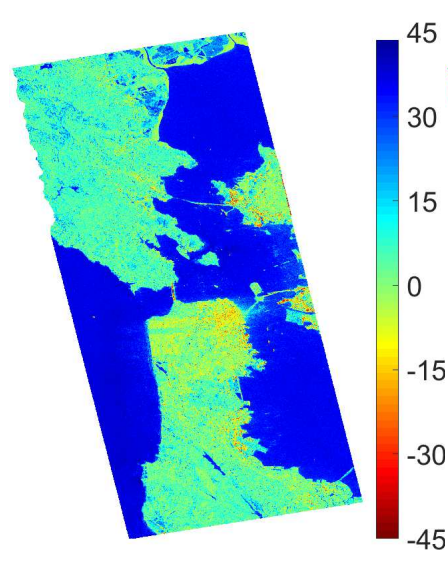

(a) $\bar{\alpha}$

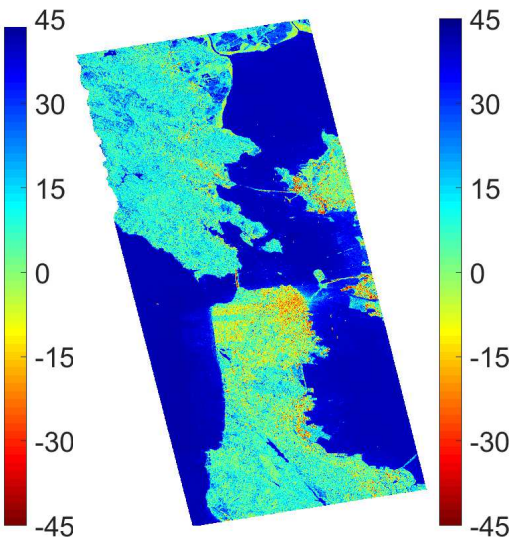

(b) $\theta_{\mathrm{FP}}$
Fig. 3. Images of $\bar{\alpha}$ and $\theta_{\mathrm{FP}}$ for RS-2 FP SAR data over San Francisco.

Figures 5 and 6 show histograms of $\bar{\alpha}, \theta_{\mathrm{FP}}$ and $m_{\mathrm{FP}}$, along with their notched boxplots (the notches are approximate confidence intervals for the median at $95 \%$ ), over selected areas of FP images, identified as "U", "O", and "F" / "OU" in Fig. 1. In the ALOS-2 FP data, "U" denotes the urban area, "O" denotes the ocean area, and " $F$ " denotes the forest

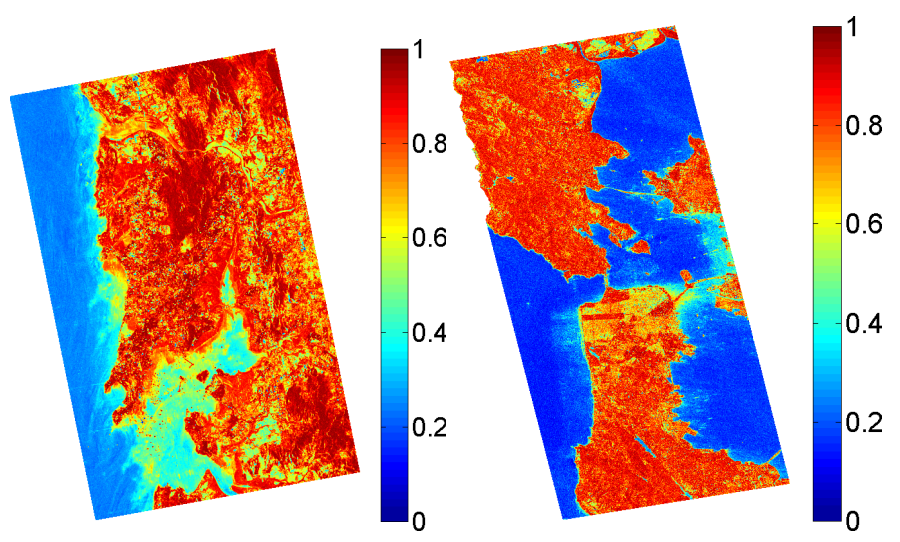

(a)

(b)

Fig. 4. Images of $H$ for (a) ALOS-2 FP and (b) RS-2 FP SAR data over Mumbai and San Francisco, respectively. 
area; while in the RS-2 FP data, " $U$ " denotes urban area, " $O$ " denotes ocean area and "OU" denotes the oriented urban area.

1) Comparison of $\theta_{\mathrm{FP}}$ with $\bar{\alpha}$ : Figures 5 and 6 show that both $\bar{\alpha}$ and $\theta_{\mathrm{FP}}$ follow a similar trend over the land-cover types which indicates comparable behavior for characterizing scattering-types from different targets.

Over the region "O", the ocean area of ALOS-2 FP data, (Fig. 5), the 3D Barakat degree of polarization $\left(m_{\mathrm{FP}}\right)$ varies between 0.95 and 1 , and both $\theta_{\mathrm{FP}}$ and $\bar{\alpha}$ are +ve valued. Besides this, we can observe in Fig. 4 that the values of $H$ over this region is also low ( 0.1 to 0.3$)$. In general, when the ocean surface is smooth, co-polarized backscatter signatures show a low coefficient of variation, which is associated with a high $3 \mathrm{D}$ Barakat degree of polarization [36]. Alongside this, over the ocean surface, an odd-bounce scattering mechanism dominates due to which both $\theta_{\mathrm{FP}}$ and $\bar{\alpha}$ show high +ve values. However, the value of $\bar{\alpha}$ is roughly between $27^{\circ}$ to $29^{\circ}$, whereas the value of $\theta_{\mathrm{FP}}$ is around $35^{\circ}$ to $37^{\circ}$. This indicates that $\theta_{\mathrm{FP}}$ is able to better characterize purer odd-bounce scattering mechanism than $\bar{\alpha}$.

Similarly to $m_{\mathrm{FP}}$ over "O", the value of $m_{\mathrm{FP}}$ over " $\mathrm{U}$ " is also high. However, the values of $\bar{\alpha}$ and $\theta_{\mathrm{FP}}$ lead to infer the presence of even-bounce scattering from these urban areas. Besides, the value of $\theta_{\mathrm{FP}}$ is around $8^{\circ}$ to $12^{\circ}$ higher than $\bar{\alpha}$. In contrast, the value of $m_{\mathrm{FP}}$ over " $\mathrm{F}$ " is low, which might be due to multiple scattering of the EM wave with distributed targets over moderately dense vegetated areas [37]. The value of $m_{\mathrm{FP}}$ is around 0.3 to 0.6 over the vegetation area, which suggests a certain amount of polarization structure in the wave. This can be attributed to small fluctuations of $\theta_{\mathrm{FP}}$ in the range $-8.0^{\circ}$ to $12.0^{\circ}$ over this region as seen in Fig. 5 .

Fig. 6 shows a comparison between $\bar{\alpha}$ and $\theta_{\mathrm{FP}}$ for the $\mathrm{C}$ band RS-2 image over San Francisco and Fig. 4 (b) shows the spatial distribution of $H$ over the same region. The behavior of the data suggests a higher discriminating power of $\theta_{\mathrm{FP}}$ over $\bar{\alpha}$. Similarly to the ALOS2 FP data, Fig. 6 shows high values of $m_{\mathrm{FP}}$ over " $\mathrm{O}$ " in ocean surface. It is known that odd-bounce scattering dominates over the ocean surface; this is confirmed by values of $\theta_{\mathrm{FP}} \approx 42^{\circ}$, which are higher than $\bar{\alpha} \approx 38^{\circ}$. Hence, $\theta_{\mathrm{FP}}$ indicates a purer scattering-type than $\bar{\alpha}$.

The "OU" region is an urban area that is oriented obliquely about the radar line of sight. This orientation contributes a strong cross-polarization component [38], [39] which decreases the value of $m_{\mathrm{FP}}$ and increases the values of $H$ over this region as shown in Fig. 4. However, the values of $\theta_{\mathrm{FP}}$ indicate the presence of even-bounce scatterers better than $\bar{\alpha}$ by $\approx 6^{\circ}$. Over the orthogonal urban area, "U", the range of $\theta_{\mathrm{FP}}$ is $\approx-18^{\circ}$ to $-23^{\circ}$, while the range of $\bar{\alpha}$ is $\approx-5^{\circ}$ to $-12^{\circ}$. Over this area, $m_{\mathrm{FP}}$ ranges between 0.78 and 0.82 . Also, substantial contributions of odd-bounce scattering component in this area influenced the ranges of both $\theta_{\mathrm{FP}}$ and $\bar{\alpha}$.

Hence, it is noteworthy that, for both the L- and C-band SAR images, $\theta_{\mathrm{FP}}$ can extract more information from the scattered wave component and, thus, it is more useful for enhanced target characterization than $\bar{\alpha}$. This improved ability is likely to be due to the joint utilization of 3D Barakat degree of polarization and received wave information in $\theta_{\mathrm{FP}}$.

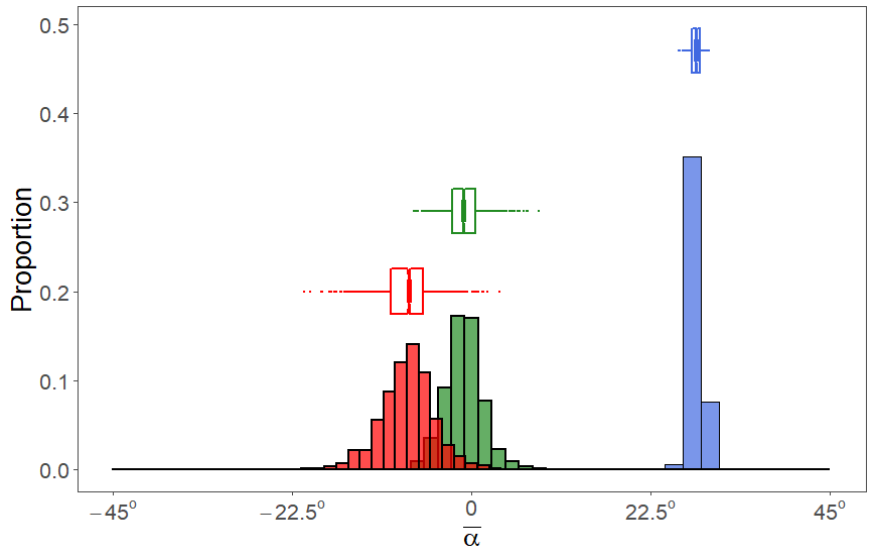

(a) $\bar{\alpha}$

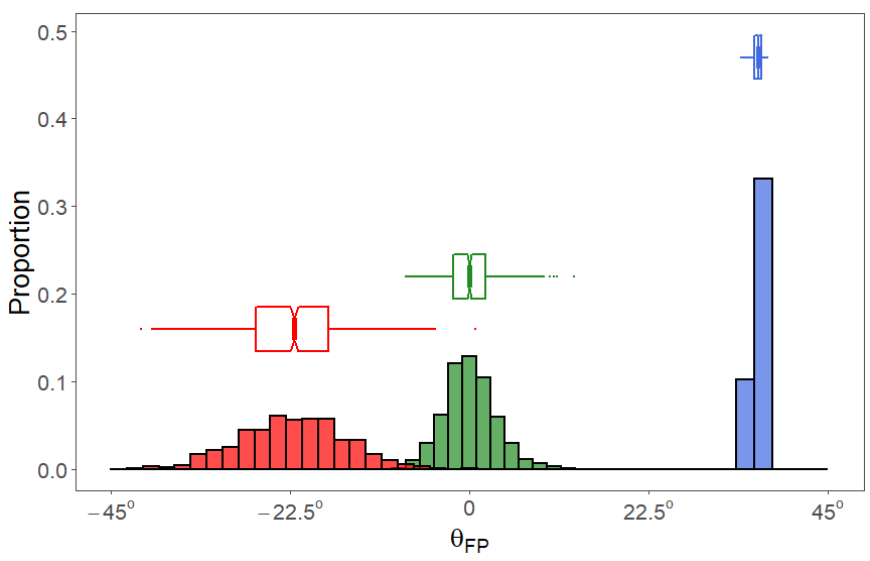

(b) $\theta_{\mathrm{FP}}$

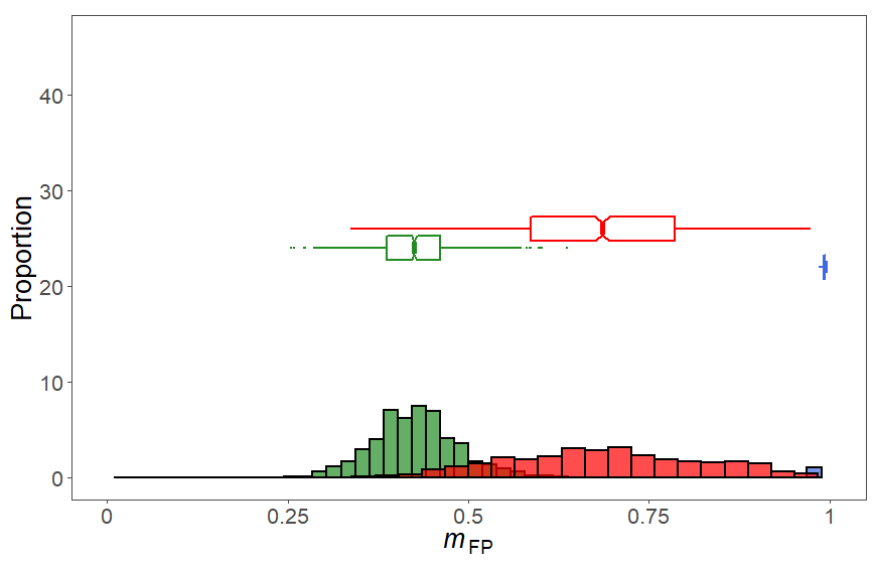

(c) $m_{\mathrm{FP}}$

Fig. 5. Comparison of $\theta_{\mathrm{FP}}$ with $\bar{\alpha}$ and $m_{\mathrm{FP}}$ for "U" (red), "F" (green) and "O" (blue) over FP ALOS-2 L-band data.

2) Decomposed power components: We use the proposed target scattering-type parameter $\theta_{\mathrm{FP}}$ to develop a new threecomponent scattering power decomposition technique, as given in Eqs. (6), (7), and (8). We compare the even-bounce $\left(P_{d}^{\mathrm{FP}}\right)$, diffused $\left(P_{v}^{\mathrm{FP}}\right)$, and odd-bounce $\left(P_{s}^{\mathrm{FP}}\right)$ scattering power components for (i) the proposed technique and the evenbounce, odd-bounce and volume scattering powers for the (ii) Freeman-Durden three-component scattering power decomposition (F3D) and the (ii) Yamaguchi four-component 


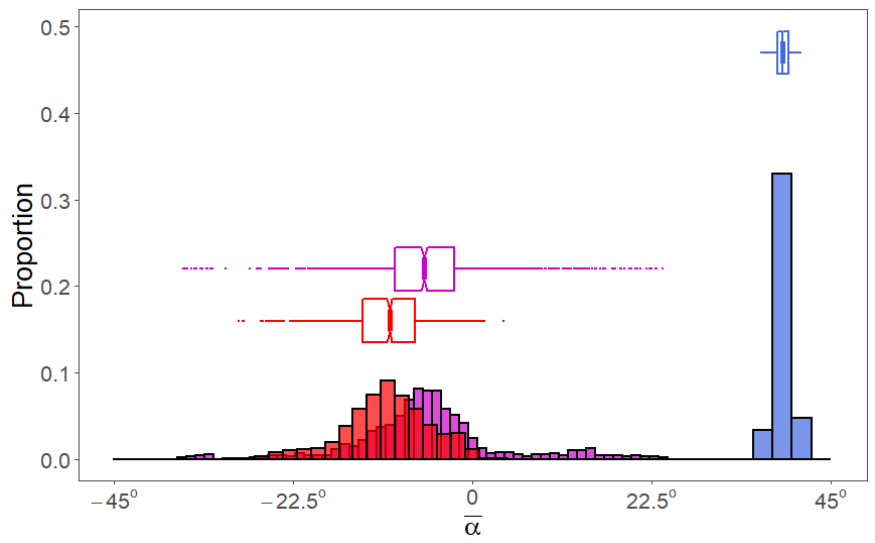

(a) $\bar{\alpha}$

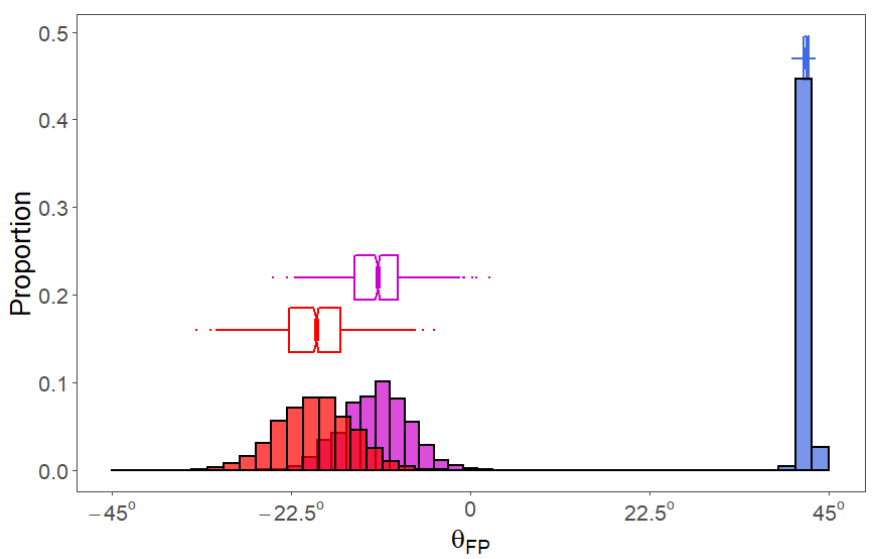

(b) $\theta_{\mathrm{FP}}$

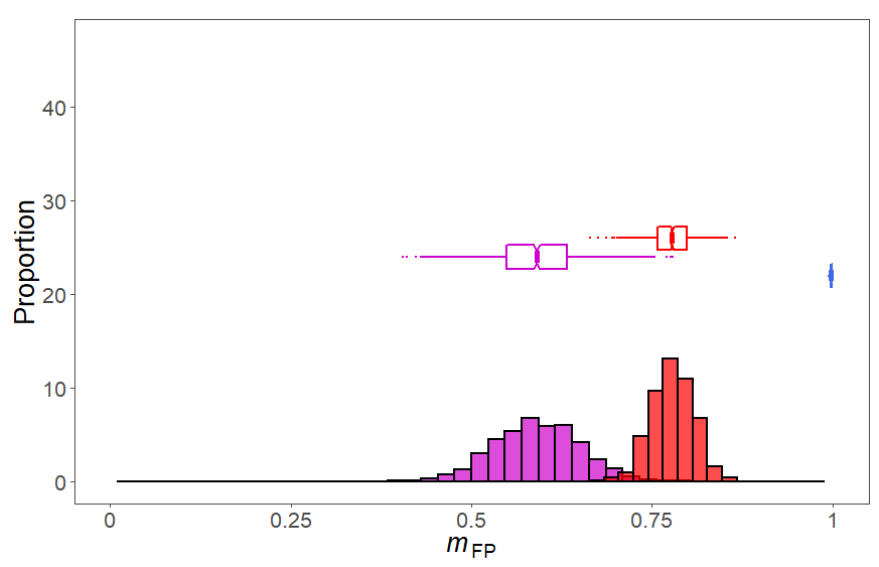

(c) $m_{\mathrm{FP}}$

Fig. 6. Comparison of $\theta_{\mathrm{FP}}$ with $\bar{\alpha}$ and $m_{\mathrm{FP}}$ for "U" (red), "OU" (magenta) and "O" (blue) over FP RS-2 C-band data.

model-based decomposition (Y4R), obtained with the L-band ALOS-2 Mumbai images for different scattering targets.

The dominant odd-bounce scattering power is apparent from the three decompositions over the ocean surface ("O") while the entropy is also low ( $H \approx 0.1$ to 0.3 ) as compared to other scattering targets as seen in Fig. 4. Here, the sample mean values of $P_{s}^{\mathrm{FP}}$ are $-11.35 \mathrm{~dB}$ and $-11.36 \mathrm{~dB}$ for $\mathrm{F} 3 \mathrm{D}$ and Y4R, respectively, whereas the mean value is $-11.11 \mathrm{~dB}$ for the proposed decomposition. This shows an increase of the odd-bounce scattering power by $2 \%$ to $3 \%$ which is mainly due to the ability of the proposed technique to extract appropriate power component from pure targets.

In general, an ocean surface is moderately smooth. Hence, the fraction of the diffused scattering component should be minimal in the total scattering power. In this regard, the sample mean value of $P_{v}^{\mathrm{FP}}$ obtained from the proposed method is $-32.36 \mathrm{~dB}$, whereas the sample mean value for the volume scattering power for both F3D and Y4R is $\approx-20.73 \mathrm{~dB}$. Therefore, the proposed technique provides a significant and desired decrease $(\approx 11.63 \mathrm{~dB})$ in the value of the diffused scattering component.

On the one hand, over the urban area ("U"), the sample mean value of $P_{d}^{\mathrm{FP}}$ obtained from the proposed decomposition technique is $0.27 \mathrm{~dB}$ and $0.52 \mathrm{~dB}$ higher than the $P_{d}^{\mathrm{FP}}$ obtained from Y4R and F3D, respectively. On the other hand, the value of the diffused scattering component, $P_{v}^{\mathrm{FP}}$ over the forest area ("F"), is $0.4 \mathrm{~dB}$ and $0.6 \mathrm{~dB}$ lower than the volume scattering powers for Y4R and F3D, respectively. Moreover, Fig. 7 shows an increase in the even-bounce scattering power over the forest area ("F"). Ref. [20] reports a similar result from a forest area. Thus, the inclusion of the 3D Barakat degree of polarization is useful to obtain the desired results from diverse targets.

Fig. 7 shows the results of applying the three decomposition techniques to the ALOS-2 image over Mumbai. Figures 7a, 7b, and 7c show, respectively, the images produced by F3D, Y4R, and the proposed decomposition technique. Figure 7 shows the percentage of the power components over urban, ocean, and forest areas, respectively, in order to provide a qualitative analysis of these results.

Over the urban area the proposed technique shows an evenbounce scattering power of $83.5 \%$ while the even-bounce scattering power is $78.6 \%$ for $\mathrm{F} 3 \mathrm{D}$ and $79.1 \%$ for Y4R. In contrast, the diffused power component is $1.1 \%$ for the proposed technique, while the volume scattering powers are $7.6 \%$ and $2.9 \%$ for F3D and Y4R, respectively. These results are illustrated in Fig. 7d, Fig. 7e and Fig. 7f.

The proposed technique increases the odd-bounce scattering power over the ocean by $13.4 \%$ with respect to $\mathrm{F} 3 \mathrm{D}$, and by $7.9 \%$ with respect to $\mathrm{Y} 4 \mathrm{R}$. Figures $7 \mathrm{~g}, 7 \mathrm{~h}$, and $7 \mathrm{i}$ illustrate these results.

On the other hand, over the forest area, "F", the proposed technique decreases the diffused scattering power by $7.3 \%$ with respect to the volume scattering power for $\mathrm{F} 3 \mathrm{D}$, and by $3 \%$ with respect to Y4R, which are evident from Figs. $71,7 \mathrm{j}$ and $7 \mathrm{k}$, respectively.

In general, these differences between the diffused and the volume scattering powers might be due to the utilization of different volume scattering models in model-based decomposition techniques. Besides, an increase in even-bounce power is also evident in the plot. This might be because of the ability of the L-band wave to penetrate the vegetation canopy and interact with the ground and trunks. It may be noted that Ref. [20] also addressed a similar increase in even-bounce power.

Fig. 8 shows the comparison of the even-bounce $\left(P_{d}^{\mathrm{FP}}\right)$, diffused $\left(P_{v}^{\mathrm{FP}}\right)$, and odd-bounce $\left(P_{s}^{\mathrm{FP}}\right)$ scattering power com- 


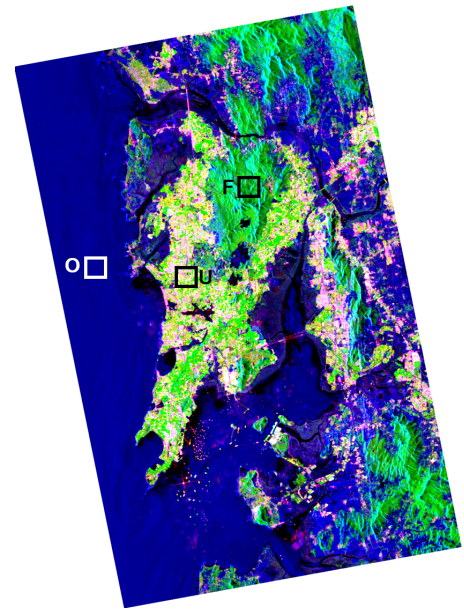

(a) F3D

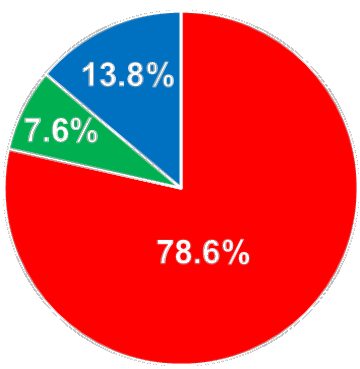

(d) F3D: U

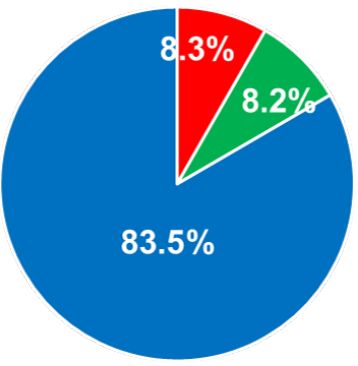

(g) F3D: O

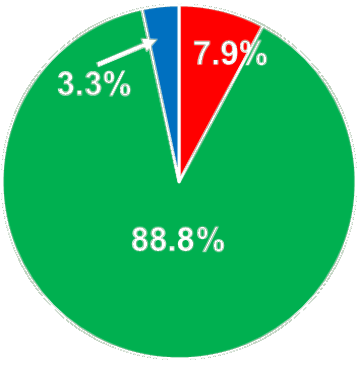

(j) F3D: F

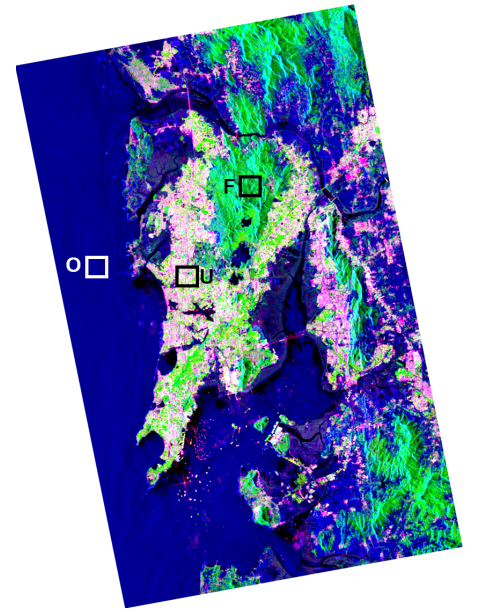

(b) $\mathrm{Y} 4 \mathrm{R}$

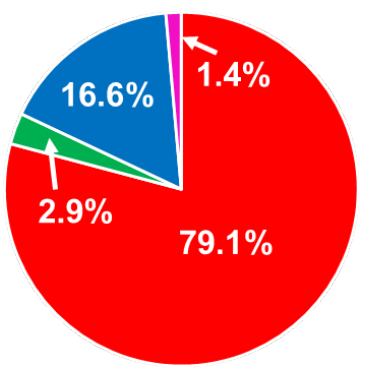

(e) Y4R: U

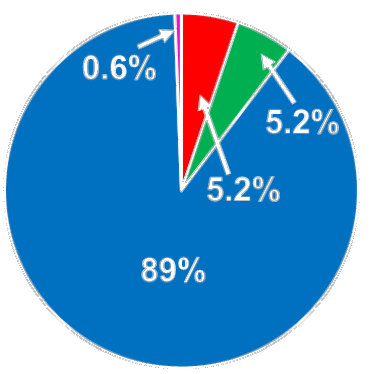

(h) Y4R: O

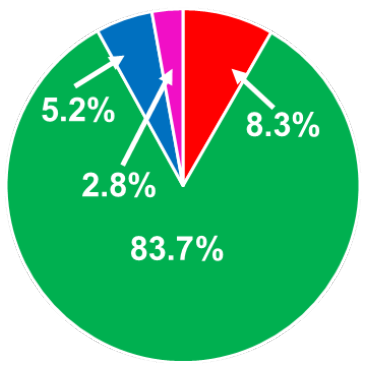

(k) Y4R: F

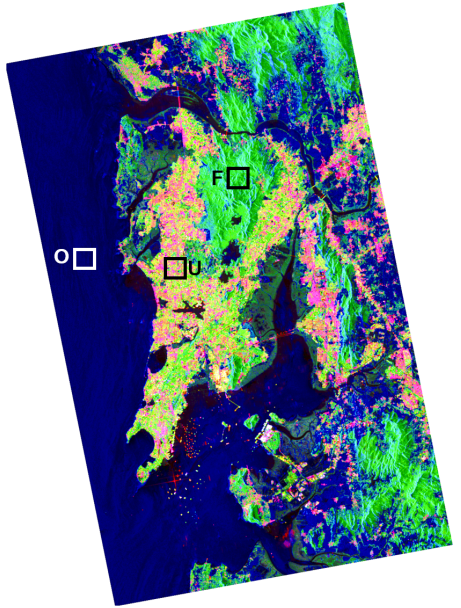

(c) Proposed

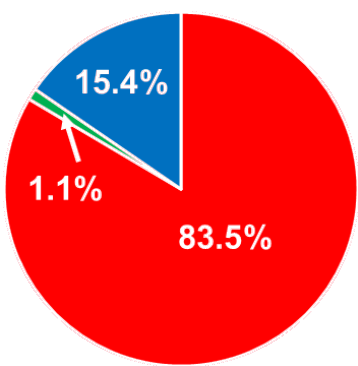

(f) Proposed: U

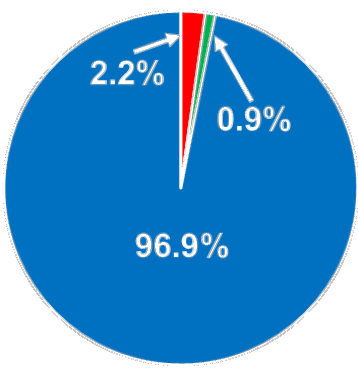

(i) Proposed: O

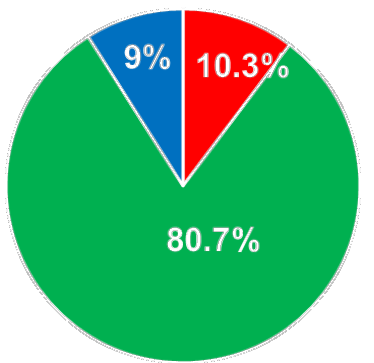

(1) Proposed: F

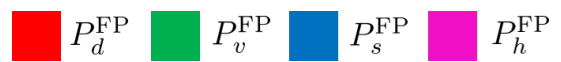

Fig. 7. Comparison of the proposed full-pol decomposition powers with Freeman (F3D) and Yamaguchi 4-component decomposition with rotation (Y4R) powers over different areas for full polarimetric L-band ALOS-2 SAR data over Mumbai, India.

ponents for the proposed technique and the powers from F3D and Y4R for the C-band RS-2 San-Francisco image.

Over the ocean surface ("O"), the value of $H$ is low and the dominant odd-bounce scattering power is apparent from the three decompositions. Here, the proposed technique shows increased odd-bounce power by $\approx 0.16 \mathrm{~dB}$ and $\approx 0.18 \mathrm{~dB}$ as compared to the Y4R and F3D decompositions, respectively. The increase in the even-bounce scattering power over the 
rotated urban area ("OU") is noteworthy. The even-bounce scattering power for the proposed technique is $\approx 1.65 \mathrm{~dB}$ higher than $\mathrm{F} 3 \mathrm{D}$, and $\approx 1.15 \mathrm{~dB}$ higher than $\mathrm{Y} 4 \mathrm{R}$. Even though $m_{\mathrm{FP}}$ varies between 0.3 and 0.6 over the rotated urban area, the value of $\theta_{\mathrm{FP}}$ can suitably characterize it as evenbounce scattering. Fig. 3 corroborates these observations.

Over the urban area ("U"), the sample mean of the evenbounce scattering power, $P_{d}^{\mathrm{FP}}$, has increased by $0.3 \mathrm{~dB}$ and $0.15 \mathrm{~dB}$, as compared to F3D and Y4R, respectively. Besides, the diffused scattering powers have decreased over this area. A similar result can also be seen in the ALOS-2 Mumbai image.

Fig. 8 shows the decomposed scattering power images, along with the percentage of the scattering power components over the San Francisco C-band Radarsat-2 image.

The volume scattering component dominates over the rotated urban area ("OU") according to F3D and Y4R: its power is $61.2 \%$ for $\mathrm{F} 3 \mathrm{D}$ and $51.6 \%$ for Y4R. In contrast, the proposed technique correctly shows dominant even-bounce scattering power of $55.5 \%$ and a diffused scattering power of $34.2 \%$. However, differences in even-bounce power between "U" and "OU" is due to variations in scattering randomness which can be observed in Fig. 4. Hence, the proposed technique enhances the even-bounce scattering power by approximately $13 \%$ and $18 \%$ compared to F3D and Y4R, respectively.

Over the ocean area, an increase of odd-bounce scattering power by $\approx 4 \%$ is apparent from the plots. As discussed earlier, the diffused scattering power over the urban area has decreased by $\approx 7.3 \%$ from F3D and $\approx 0.7 \%$ from $\mathrm{Y} 4 \mathrm{R}$. The odd-bounce scattering power has decreased by $\approx 2 \%$ to $5 \%$ from F3D and Y4R, respectively. Besides this, the helix power component $\left(P_{h}^{\mathrm{FP}}\right)$ obtained from the $\mathrm{Y} 4 \mathrm{R}$ decomposition is negligible for most of the targets as compared to the other three scattering power components. Therefore, the proposed technique ascertains its ability to extract pertinent information about coherent targets and better characterizes roll-invariant targets from fully polarimetric SAR data.

\section{B. Compact polarimetry}

We simulated hybrid-compact polarimetric SAR data from the ALOS-2 L-band FP SAR data over Mumbai and from the RS-2 C-band FP SAR data over San Francisco. We obtained the $2 \times 2$ covariance matrices $\mathbf{C}_{2}$ from the $3 \times 3$ covariance matrices of FP data as a function of the transmitting ellipticity $\chi$ and orientation angle $\psi$. In this study, we simulated $\mathbf{C}_{2}$ with the assumption of right circular polarization on transmit, i.e., $\chi=-45^{\circ}$ and $\psi=0^{\circ}$ [40]. This particular configuration simulates compact-pol data for a perfect circular polarization on transmit.

We compare the target scattering-type parameter, $\theta_{\mathrm{CP}}$ with the ellipticity parameter $\bar{\chi}$ over the same areas used for the analysis of the FP images. Similarly to $\theta_{\mathrm{FP}}$, the values of $\theta_{\mathrm{CP}}$ and $\bar{\chi}$ vary from $-45^{\circ}$ to $45^{\circ}$ and, thus, are comparable. $\theta_{\mathrm{CP}}=-45^{\circ}$ for pure even-bounce scattering and $\theta_{\mathrm{CP}}=45^{\circ}$ for pure odd-bounce scattering, whereas $\theta_{\mathrm{CP}}=0^{\circ}$ for diffused scattering.
Figs. 9 and 11 show $\bar{\chi}$ and $\theta_{\mathrm{CP}}$ over Mumbai and San Francisco, respectively. Both parameters span from odd-bounce scattering to even-bounce scattering characteristics.

1) Comparison of $\theta_{\mathrm{CP}}$ with $\bar{\chi}$ : Fig. 12 shows histograms and notched boxplots of $\theta_{\mathrm{CP}}$ and $\bar{\chi}$ over the ALOS-2 L-band image. The difference between $\theta_{\mathrm{CP}}$ and $\bar{\chi}$ are most noticeable in two regions: (1) region " $\mathrm{O}$ ", in blue, which is over the ocean surface, and (2) region " $F$ ", in green, which is over a forested area. In region "O", the value of $\bar{\chi}$ varies from $\approx 30^{\circ}$ to $32^{\circ}$ whereas, $\theta_{\mathrm{CP}}$ varies from $\approx 37^{\circ}$ to $39^{\circ}$. Moreover, the value of $m_{\mathrm{CP}}$ is high in the region "O", which confirms that the EM wave is majorly polarized in this region.

Over the region " $F$ ", the value of $m_{\mathrm{CP}}$ varies from $\approx 0.07$ to 0.15 , indicating low polarization due to random scattering. Here, $\theta_{\mathrm{CP}}$ fluctuates around $0.5^{\circ}$, while $\bar{\chi}$ varies from $\approx-18^{\circ}$ to $12^{\circ}$. Therefore, similarly to $\theta_{\mathrm{FP}}, \theta_{\mathrm{CP}}$ suitably characterizes distributed targets than $\bar{\chi}$ due to its better consistency.

Fig. 13 shows similar results for the C-band RS-2 compact polarimetric SAR data: ocean surface ("O") in blue, orthogonal urban ("U") in red, and rotated urban ("RU") in magenta. Over the region " $\mathrm{O}$ ", the value of $\theta_{\mathrm{CP}}$ is $\approx 3^{\circ}$ to $5^{\circ}$ higher than $\bar{\chi}$. Therefore, the performance of $\theta_{\mathrm{CP}}$ is better than $\bar{\chi}$ for the ocean area. Furthermore, $\theta_{\mathrm{CP}}$ fluctuates around $-21^{\circ}$ over "U", and $-13^{\circ}$ over "OU", whereas, the variation of $\bar{\chi}$ is similar over the region " $U$ ".

2) Decomposed power components: Fig. 14 shows the results of the scattering powers for the L-band ALOS-2 SAR data using the $S-\Omega, m-\chi$, and the proposed technique. Overall, we can notice that the results of the proposed technique are better than $S-\Omega$ and $m-\chi$ decomposition techniques. The $P_{s}^{C P}$ power is marginally higher than $S-\Omega$ and $m$ - $\chi$ over the ocean region "O". However, we can notice a decrease of $\approx 9.5 \mathrm{~dB}$ in the $P_{d}^{\mathrm{CP}}$ power by the proposed technique compared to $S-\Omega$ over this region. In contrast, the difference in the even-bounce power between $m-\chi$, and the proposed technique is of $6.13 \mathrm{~dB}$.

The value of $P_{d}^{\mathrm{CP}}$ for both $S-\Omega$ and $m-\chi$ is $\approx 7.03 \mathrm{~dB}$ over the urban area "U", whereas, $P_{d}^{C P}$ is $\approx 7.47 \mathrm{~dB}$ for the proposed technique. Therefore, the proposed technique discriminates better odd-bounce and even-bounce scattering than $S-\Omega$ and $m-\chi$.

$P_{v}^{\mathrm{CP}} \approx-6.54 \mathrm{~dB}$ for $S-\Omega$ and $\approx-6.35 \mathrm{~dB}$ for both $m-\chi$ and the proposed technique over the forest area. Moreover, we can notice that the diffused power for both $m$ - $\chi$, and the proposed technique are identical for target areas in the scene. This is because both $m-\chi$ and the proposed technique use the depolarization fraction $\left(1-m_{\mathrm{CP}}\right)$ of the total power to compute the diffused scattering power component. However, the presence of small, even-bounce and odd-bounce scattering powers in the forest area might be due to specific structural effects and the ability of the L-band SAR wave to penetrate the forest canopy.

We notice from the scattering power components that the dominant scattering mechanism for each area is comparable with $S-\Omega, m-\chi$, and the proposed technique. Essentially, this similarity among different techniques indicates their equivalent ability to identify dominant scatterers in the scene. However, the proposed technique provides marginally better results than the other two over urban and ocean areas for CP SAR data. 


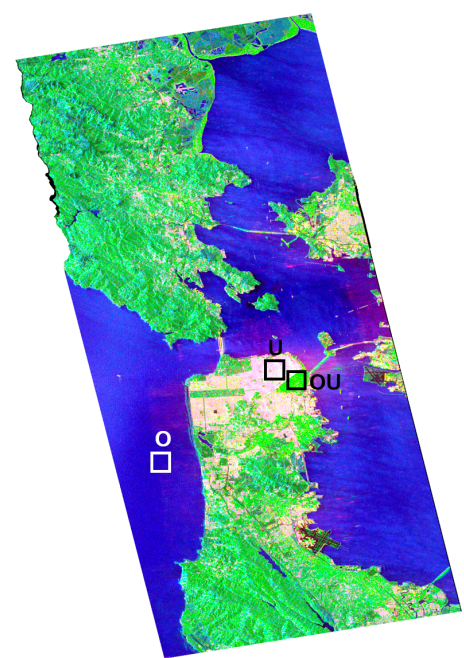

(a) F3D

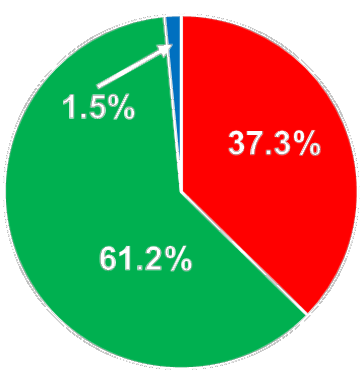

(d) F3D: OU

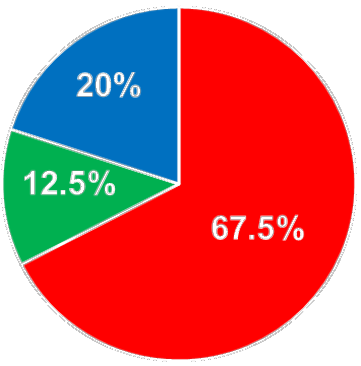

(g) F3D: U

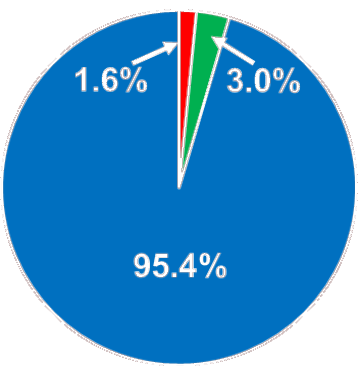

(j) F3D: O

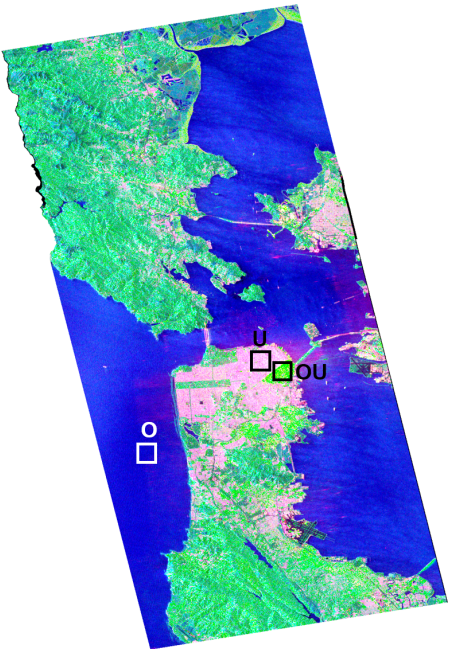

(b) $\mathrm{Y} 4 \mathrm{R}$

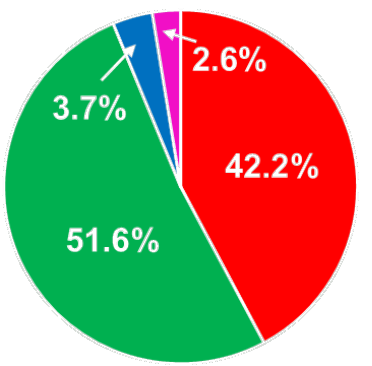

(e) Y4R: OU

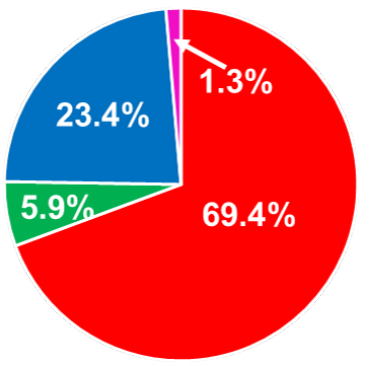

(h) Y4R: U

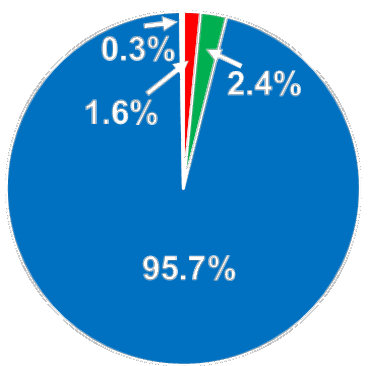

(k) Y4R: O

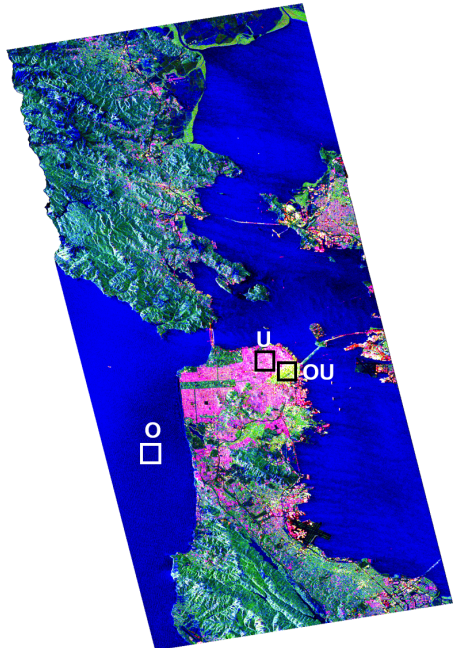

(c) Proposed

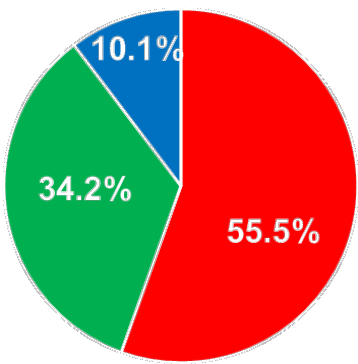

(f) Proposed: OU

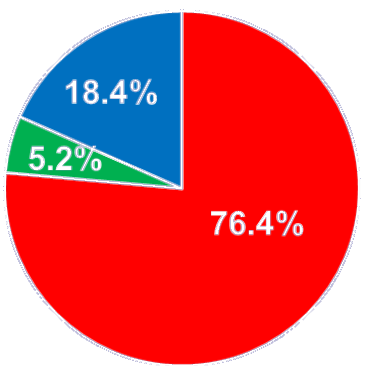

(i) Proposed: U

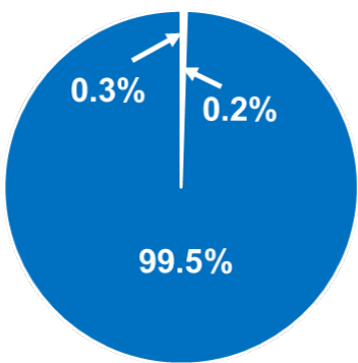

(1) Proposed: O

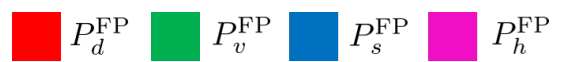

Fig. 8. Comparison of the proposed full-pol decomposition powers with Freeman (F3D) and Yamaguchi 4-component decomposition with rotation (Y4R) powers over different areas for full polarimetric C-band Radarsat-2 SAR data over San Francisco, USA.

In the following, we make quantitative assessments of threecomponent decomposition powers from $S-\Omega, m-\chi$, and the proposed technique using the C-band RS-2 compact polari- metric data. We used small patches over urban ("U"), ocean ("O"), and rotated urban ("OU") areas for comparison.

The odd-bounce scattering power over the ocean area is bet- 


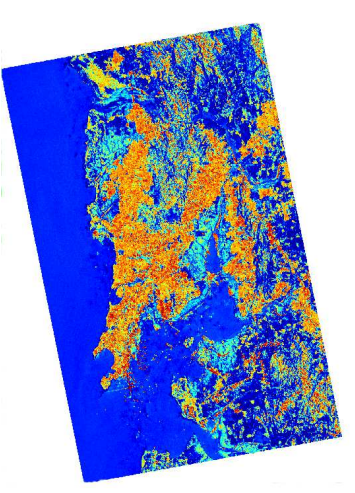

(a) $\bar{\chi}$

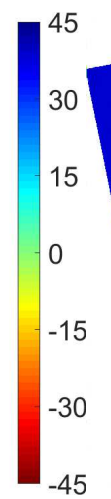

45

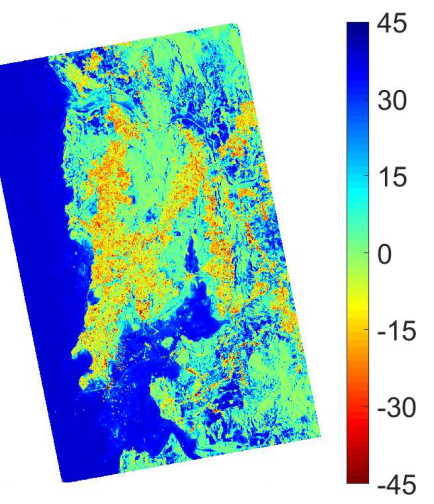

(b) $\theta_{\mathrm{CP}}$
Fig. 9. Images of $\bar{\chi}$ and $\theta_{\mathrm{CP}}$ for ALOS-2 simulated CP SAR data.

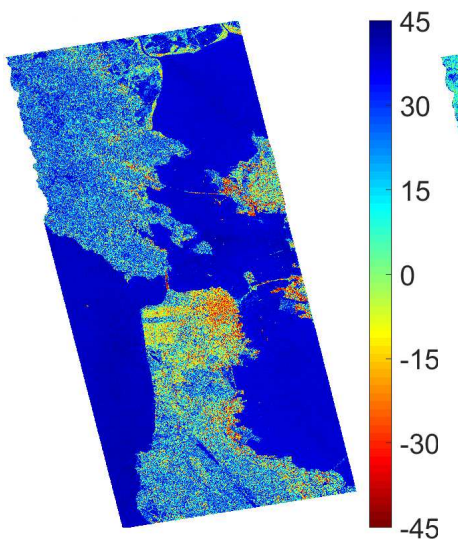

(a) $\bar{\chi}$

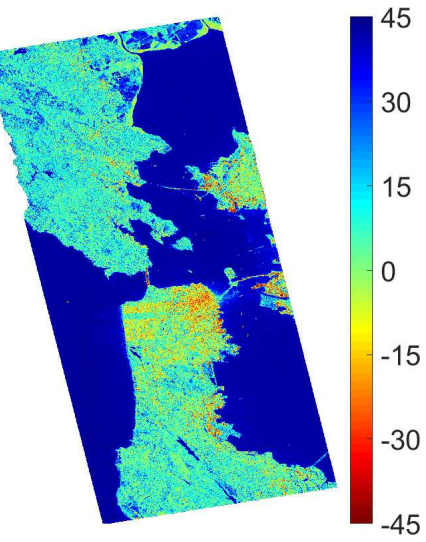

(b) $\theta_{\mathrm{CP}}$
Fig. 10. Images of $\bar{\chi}$ and $\theta_{\mathrm{CP}}$ for RS-2 simulated CP SAR data.

ter with the proposed technique than $S-\Omega$ and $m-\chi$. Besides, the even-bounce power is significantly lower by $\approx 15 \mathrm{~dB}$ and $\approx 9 \mathrm{~dB}$ for the proposed technique than $S-\Omega$ and $m-\chi$, respectively. This aspect suggests that the proposed technique adequately quantifies the dominant scattering mechanism over the ocean surface.

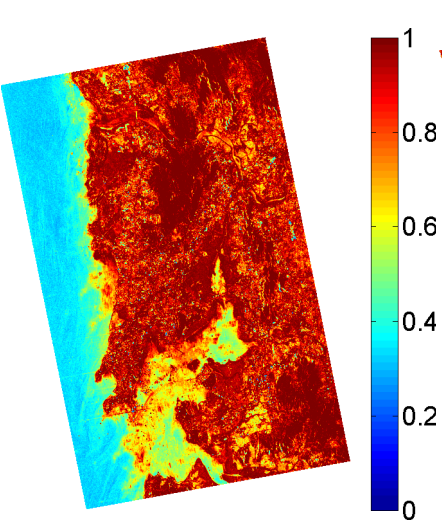

(a)

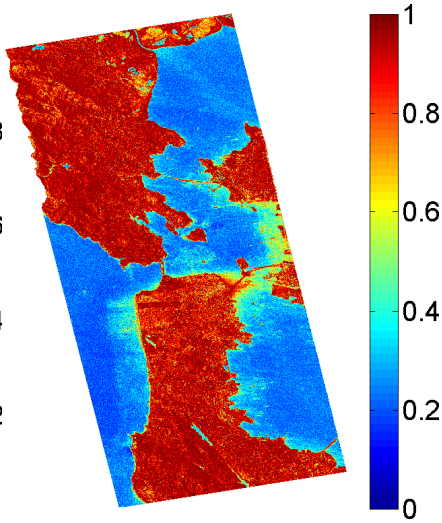

(b)
Fig. 11. Images of $H$ for (a) ALOS-2 CP and (b) RS-2 CP SAR data over Mumbai and San Francisco, respectively

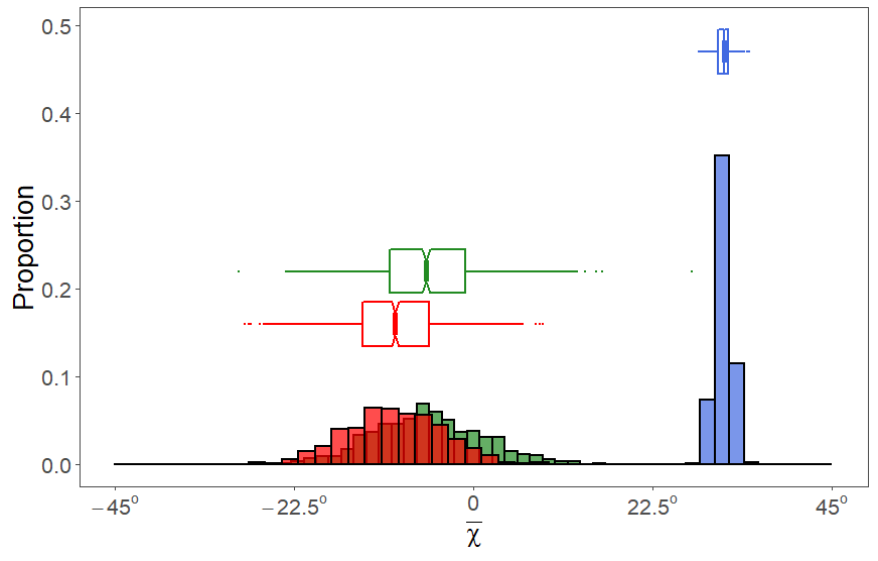

(a) $\bar{\chi}$

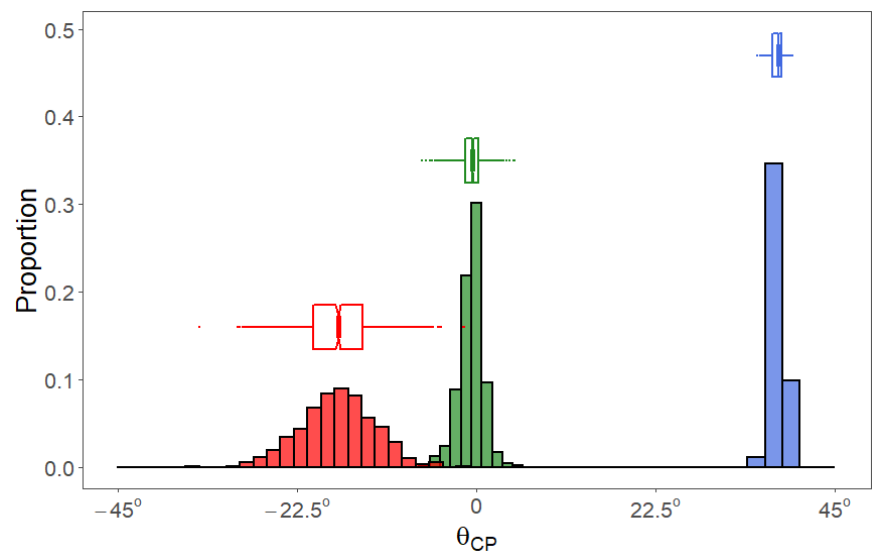

(b) $\theta_{\mathrm{CP}}$

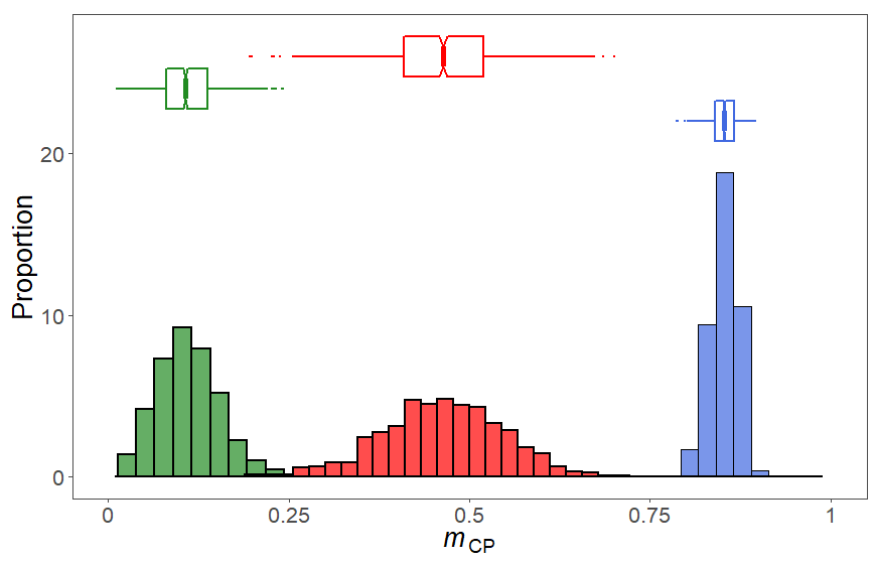

(c) $m_{\mathrm{CP}}$

Fig. 12. Comparison of $\theta_{\mathrm{CP}}$ with $\bar{\chi}$ and $m_{\mathrm{CP}}$ for urban (red), forest (green) and ocean (blue) over CP ALOS-2 L-band data.

The proposed technique increases the even-bounce scattering power over the rotated urban area. The even-bounce scattering power obtained from the proposed technique is $0.31 \mathrm{~dB}$ higher than $S-\Omega$ and $m-\chi$. However, both $m-\chi$ and the proposed technique detect small $P_{v}^{\mathrm{CP}}$ power of $-6.15 \mathrm{~dB}$ over this area. The presence of this diffused scattering power is likely due to the cross-polarization component of the EM wave generated by oriented urban areas about the radar line of sight [41], [42]. Hence, the value of $H$ over this region is 


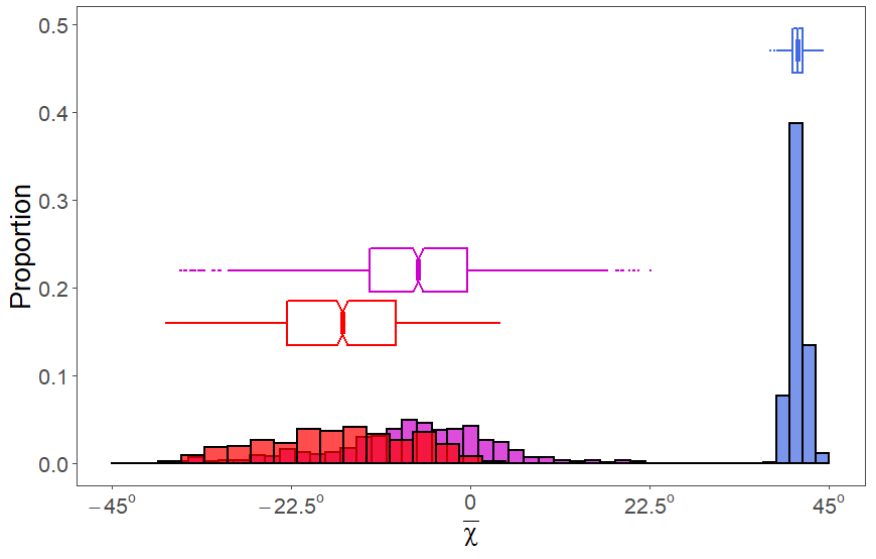

(a) $\bar{\chi}$

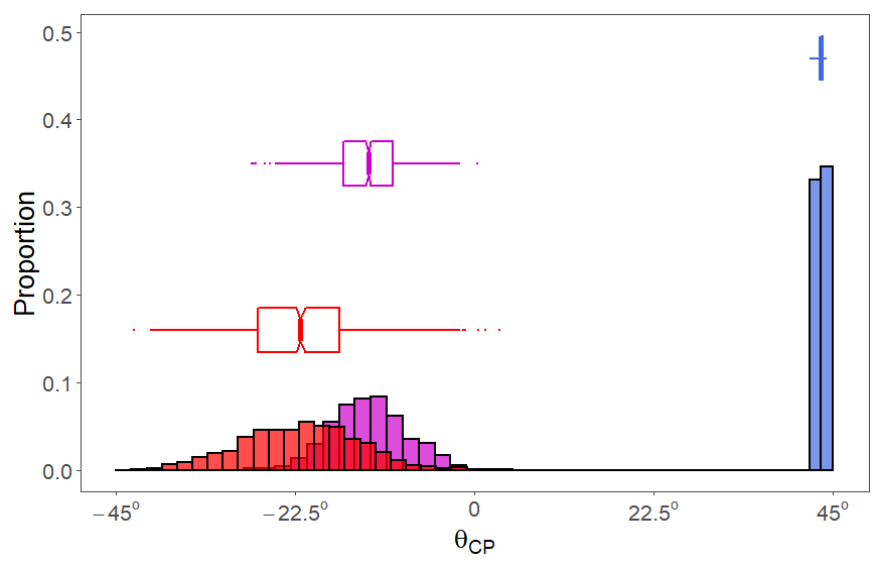

(b) $\theta_{\mathrm{CP}}$

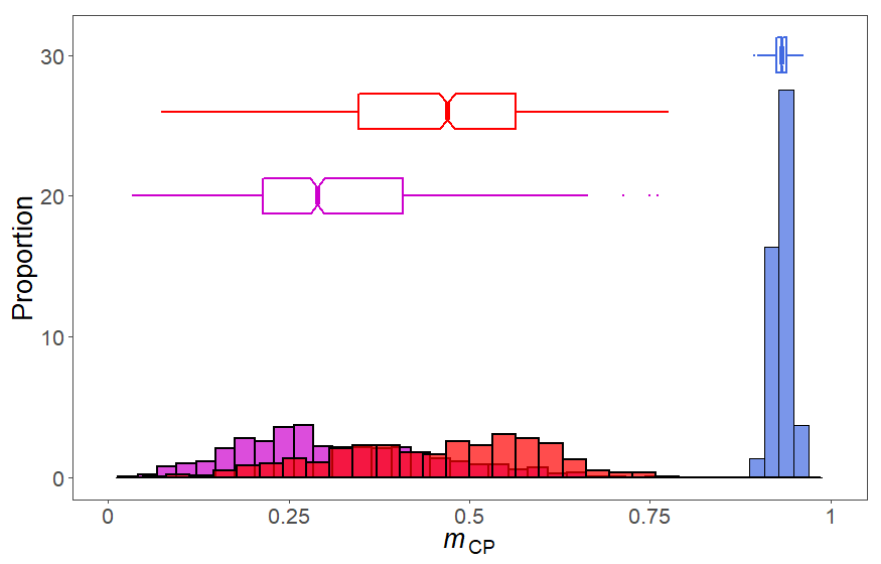

(c) $m_{\mathrm{CP}}$

Fig. 13. Comparison of $\theta_{\mathrm{CP}}$ with $\bar{\chi}$ and $m_{\mathrm{CP}}$ for urban (red), rotated urban (magenta) and ocean (blue) over CP RS-2 C-band data.

higher than the urban area by $20 \%$ to $30 \%$ as seen in Fig. 11.

Over the urban area, the even-bounce scattering power, $P_{d}^{\mathrm{CP}}$ from the proposed technique is higher than $S-\Omega$ and $m-\chi$, while $P_{d}^{C P}$ for both $m-\chi$ and $S-\Omega$ are similar. Compared to $S$ $\Omega$, we notice a $0.36 \mathrm{~dB}$ increase in the $P_{d}^{\mathrm{CP}}$ power component for the proposed technique. On the other hand, the $P_{s}^{C P}$ power is considerably lower $(\approx 2 \mathrm{~dB})$ for the proposed technique than $S-\Omega$ and than $m-\chi$. This indicates the ability of the proposed technique to quantify better the amount of pure scattering characteristics.

Fig. 15 shows the decomposed power images, along with the percentages of $P_{d}^{\mathrm{CP}}, P_{v}^{\mathrm{CP}}$, and $P_{s}^{\mathrm{CP}}$ over the rotated urban, ocean, and forest areas. All the dominant power components are similar across all the targets. Over the rotated urban area, the proposed technique retrieves around $3 \%$ more the evenbounce scattering power compared to the other methods.

In summary, it should also be noted that $S-\Omega, m-\chi$, and the proposed technique jointly use both the $2 \mathrm{D}$ Barakat degree of polarization and the received wave information in terms of the elements of the covariance matrix. This joint utilization of the wave information helps better target characterization while improving the scattering powers.

\section{CONCLUSIONS}

We proposed two unique roll-invariant scattering-type parameters: $\theta_{\mathrm{FP}}$, for full polarimetric, and $\theta_{\mathrm{CP}}$, for compact polarimetric, to characterize different targets from Synthetic Aperture Radar (SAR) data. These two parameters are derived by jointly using both 3D and 2D Barakat degree of polarization and received wave information from SAR data.

We have shown that due to the joint utilization of the 3D and 2D Barakat degree of polarization and received wave information, $\theta_{\mathrm{FP}}$ and $\theta_{\mathrm{CP}}$ can extract more purity in the EM wave as compared to $\alpha$ and $\chi$. Experimental results support that $\theta_{\mathrm{FP}}$ and $\theta_{\mathrm{CP}}$ better characterize rotated urban and vegetation areas than $\alpha$ and $\chi$. Moreover, the variability of these proposed parameters over vegetation areas is much lower than $\alpha$ and $\chi$. This aspect indicates a promising consistency of these parameters over distributed targets, similarly to even and odd-bounce targets.

The parameters are then utilized to derive non-model based three-component scattering power decomposition techniques for both FP and CP SAR data. It is noteworthy that the formulations of these two techniques are equivalent for both the SAR imaging modes. Traditional model-based decomposition techniques are limited to the optimization of the received covariance matrix while ignoring the utilization of the amount of polarization in the scattered wave. In this perspective, the use of the 3D and 2D Barakat degree of polarization has suitably enhanced the ability to discriminate distinct targets within a resolution cell. This feature is also evident from the plots of $\bar{\alpha} / \theta_{\mathrm{FP}}$ for FP data and $\bar{\chi} / \theta_{\mathrm{CP}}$ for CP data. Besides, it can be seen from the study that, the parameters, $\theta_{\mathrm{FP}}$ and $\theta_{\mathrm{CP}}$ together with the three scattering power components can adequately characterize complete scattering mechanisms from a target.

Results show that our proposed technique performs better than the Freeman-Durden three-component decomposition (F3D), Yamaguchi 4-component decomposition with rotation (Y4R) for FP data, and also better than $S-\Omega, m-\chi$ for CP data. Moreover, the proposed decomposition techniques are intuitive and model-free. Hence, the overestimation of any power component is absent, and the polarized power component has improved with the utilization of the Barakat degree of polarization. Furthermore, the proposed technique produces nonnegative power components, which is a significant drawback 


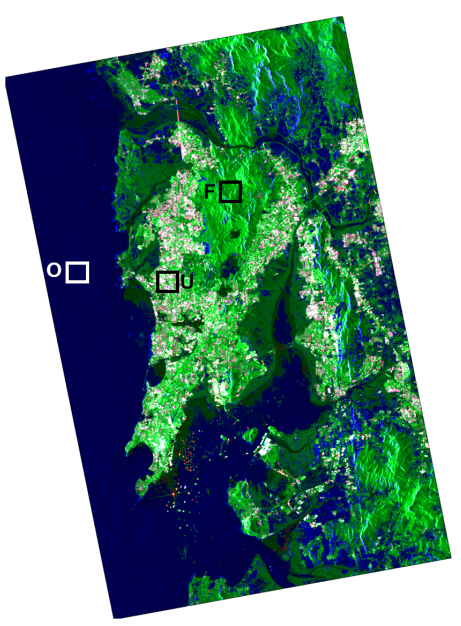

(a) $S-\Omega$

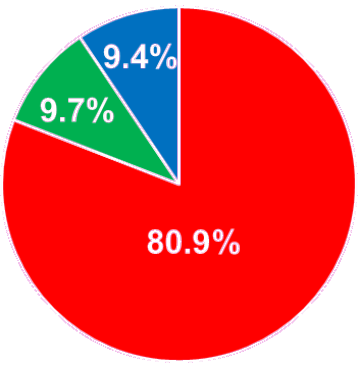

(d) $S-\Omega: \mathrm{U}$

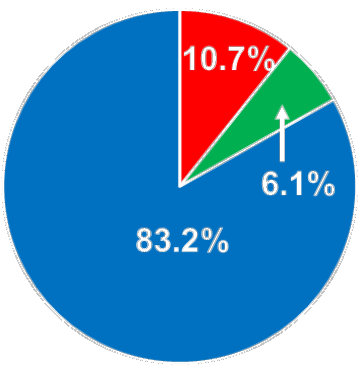

(g) $S-\Omega: \mathrm{O}$

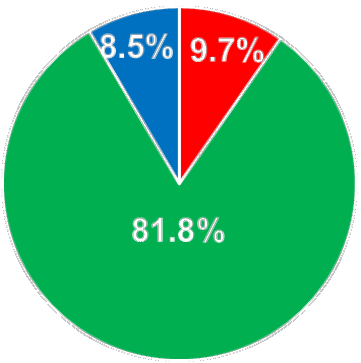

(j) $S-\Omega: \mathrm{F}$

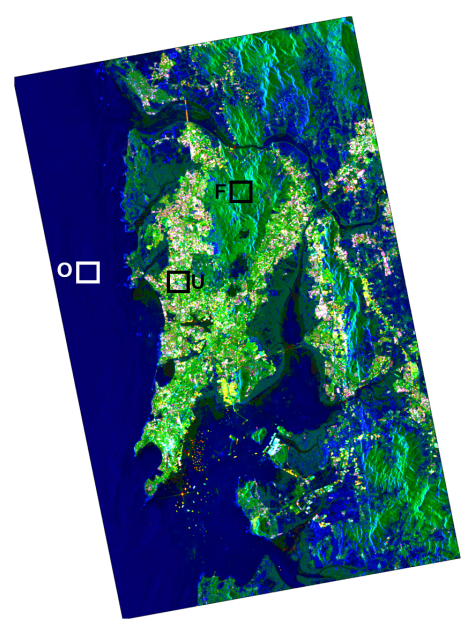

(b) $m-\chi$

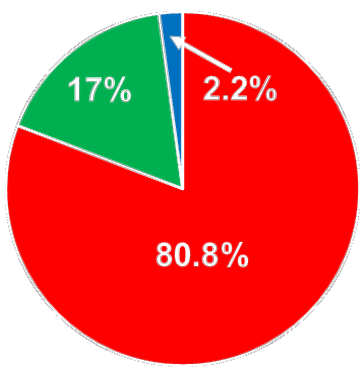

(e) $m-\chi: \mathrm{U}$

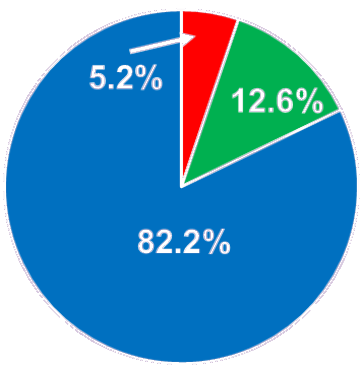

(h) $m-\chi: \mathrm{O}$

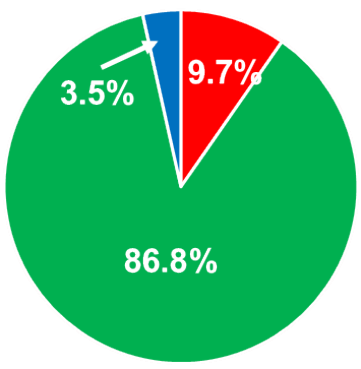

(k) $m-\chi: \mathrm{F}$

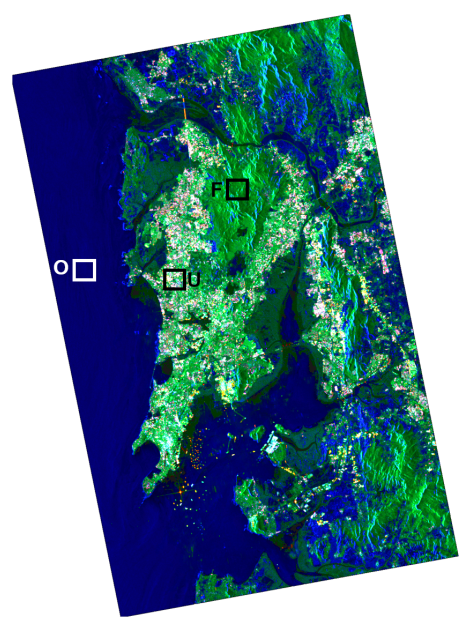

(c) Proposed

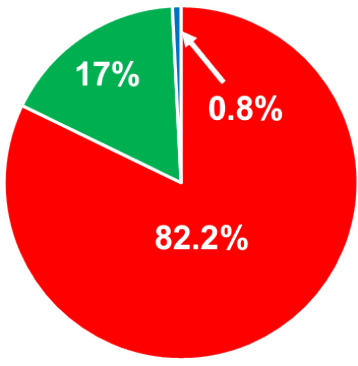

(f) Proposed: U

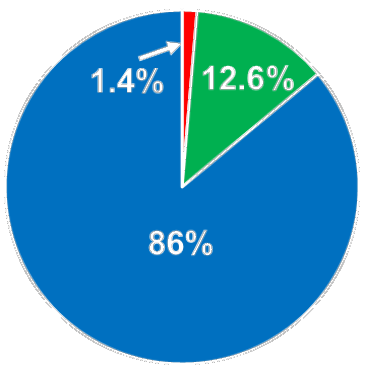

(i) Proposed: O

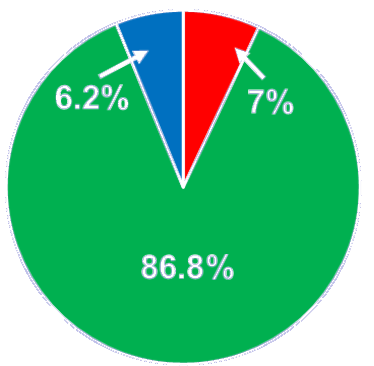

(1) Proposed: F

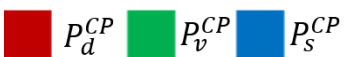

Fig. 14. Comparison of the proposed compact-pol decomposition powers with $S-\Omega$ and $m$ - $\chi$ decomposition powers over different areas for the simulated compact polarimetric L-band ALOS-2 SAR data over Mumbai, India.

of several model-based decomposition techniques, as reported in numerous studies. Moreover, the results also show the improvement of decomposed scattering powers over diverse regions.

Specifically, as addressed earlier, coherent power components have increased, which indicates the enhanced ability to 


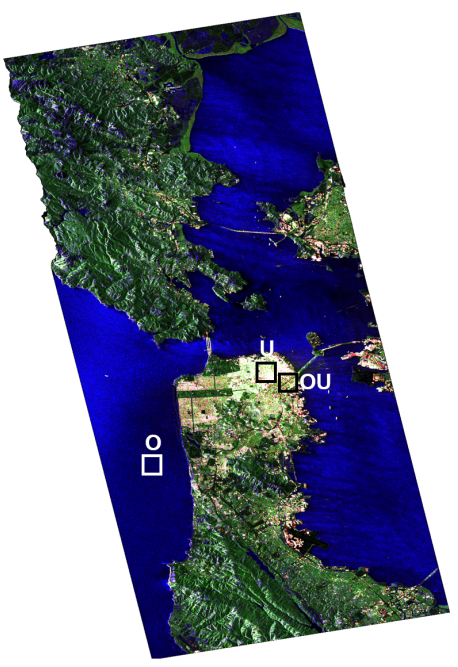

(a) $S-\Omega$

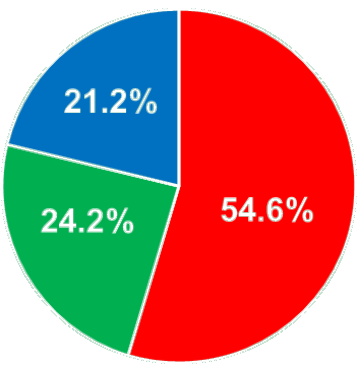

(d) $S-\Omega:$ OU

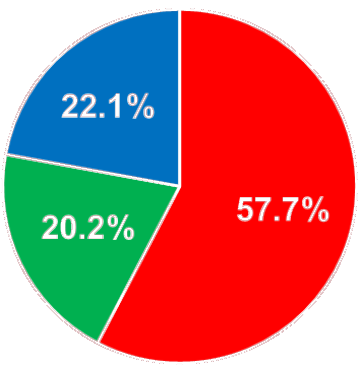

(g) $S-\Omega: \mathrm{U}$

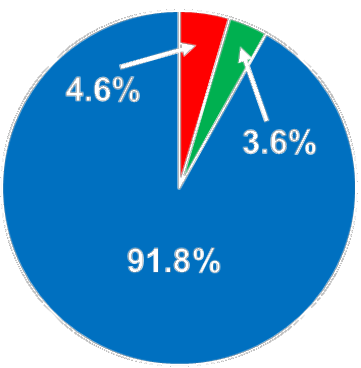

(j) $S-\Omega$ : O

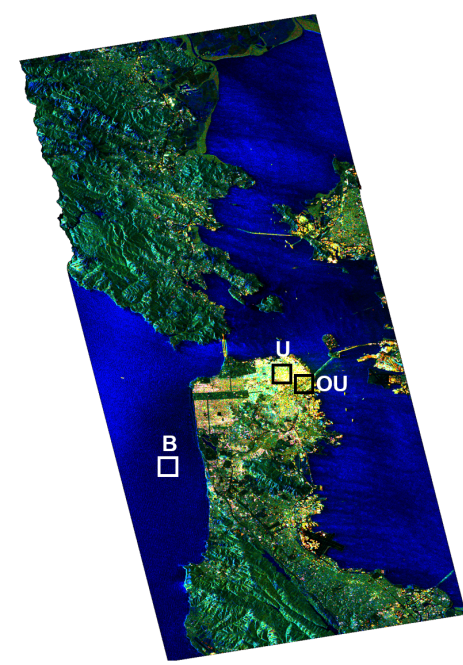

(b) $m-\chi$

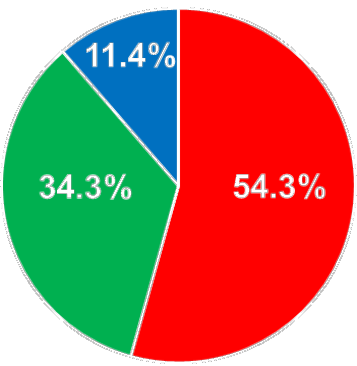

(e) $m-\chi: \mathrm{OU}$

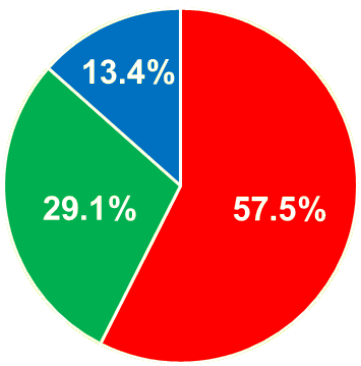

(h) $m-\chi: \mathrm{U}$

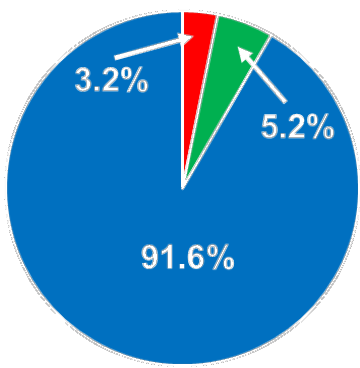

(k) $m-\chi: \mathrm{O}$

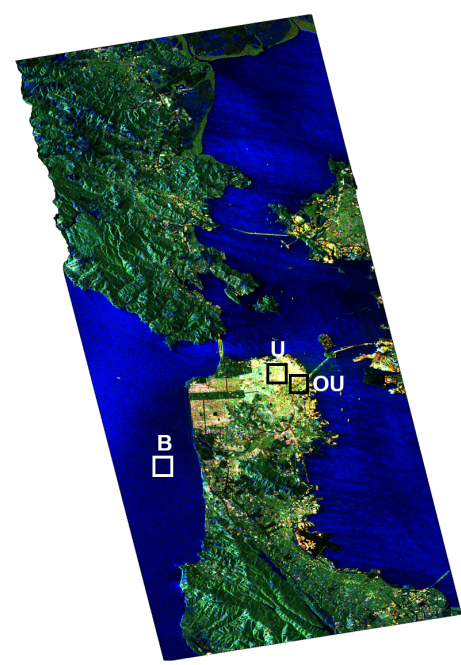

(c) Proposed

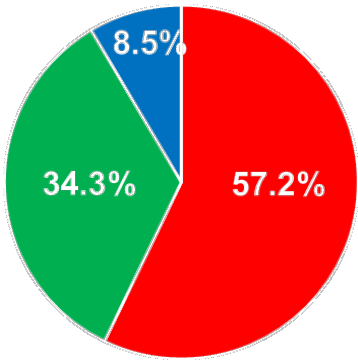

(f) Proposed: OU

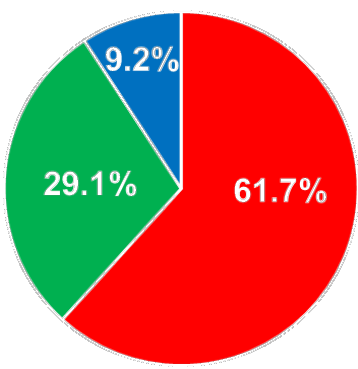

(i) Proposed: U

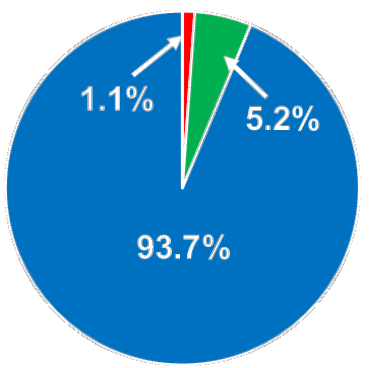

(1) Proposed: O

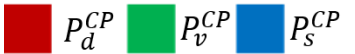

Fig. 15. Comparison of the proposed compact-pol decomposition powers with $S-\Omega$ and $m-\chi$ decomposition powers over different areas for the simulated compact polarimetric C-band RS-2 SAR data over San Francisco, USA.

extract coherent scatterers from the scene. Notably, the improvement of coherent power estimation is evident in rotated urban areas for FP data due to the roll-invariant nature of the scattering-type parameter, where both F3D and Y4R increases 
the volume power component.

The proposed technique identifies even-bounce as the dominant scattering power, which is also significantly higher than the volume component. Another essential feature is that the decomposition powers are stable, i.e., the powers are insensitive to noise, which is vital for an effective decomposition algorithm. Thus this proposed decomposition technique has excellent potential for land use and land cover analysis using both FP and CP SAR data.

\section{APPENDIX}

In this section, we prove the roll-invariant nature of $\theta_{\mathrm{FP}}$ and $\theta_{\mathrm{CP}}$, we obtain their ranges analytically, and we justify these properties from a physical perspective. We also show the relationships between $\theta_{\mathrm{FP}}$ and $\alpha$, and between $\theta_{\mathrm{CP}}$ and $\chi$.

\section{A. Range of $\theta_{\mathrm{FP}}$ and $\theta_{\mathrm{CP}}$}

1) For $\theta_{\mathrm{FP}}$ : The expression of $\theta_{\mathrm{FP}}$ is given as:

$$
\theta_{\mathrm{FP}}=\tan ^{-1}(\mathrm{~A})
$$

where,

$$
\mathrm{A}=\frac{m_{\mathrm{FP}} \operatorname{Span}\left(T_{11}-T_{22}-T_{33}\right)}{T_{11}\left(T_{22}+T_{33}\right)+m_{\mathrm{FP}}^{2} \operatorname{Span}^{2}}
$$

The range of A can be obtained by considering three coherency matrices defining the boundary curve of the feasible scattering region [1],

$$
\text { Case 1: } \mathbf{T}_{(I)}=\left[\begin{array}{lll}
1 & 0 & 0 \\
0 & \rho & 0 \\
0 & 0 & \rho
\end{array}\right] ; \quad 0 \leq \rho \leq 1
$$

The $3 \mathrm{D}$ polarization Barakat degree of polarization, $m_{\mathrm{FP}}$ is expressed in terms of the boundary parameter $\rho$ as,

$$
m_{\mathrm{FP}}=\left(1-\frac{27 \rho^{2}}{(2 \rho+1)^{3}}\right)^{\frac{1}{2}}
$$

and,

$$
\mathrm{A}=\frac{m_{\mathrm{FP}}\left(1-4 \rho^{2}\right)}{2 \rho+m_{\mathrm{FP}}^{2}\left(4 \rho^{2}+4 \rho+1\right)}
$$

Using (22) and (23) in the range of $\rho$, we get $0 \leq \mathrm{A} \leq 1$.

For the second case,

$$
\text { Case 2: } \mathbf{T}_{(I I)}=\left[\begin{array}{ccc}
2 \rho-1 & 0 & 0 \\
0 & 1 & 0 \\
0 & 0 & 1
\end{array}\right] ; \quad 0.5 \leq \rho \leq 1
$$

with,

$$
m_{\mathrm{FP}}=\left(1-\frac{27(2 \rho-1)}{(2 \rho+1)^{3}}\right)^{\frac{1}{2}}
$$

and,

$$
\mathrm{A}=\frac{m_{\mathrm{FP}}(2 \rho+1)(2 \rho-3)}{2(2 \rho-1)+m_{\mathrm{FP}}^{2}(2 \rho+1)^{2}}
$$

For the third case,

$$
\text { Case 3: } \mathbf{T}_{(I I I)}=\left[\begin{array}{ccc}
0 & 0 & 0 \\
0 & 1 & 0 \\
0 & 0 & 2 \rho
\end{array}\right] ; \quad 0.5 \leq \rho \leq 1
$$

Therefore, for, Case $3, m_{\mathrm{FP}}=1$ and $\mathrm{A}=-1$. Hence, combining the two cases (i.e., Case 2 and Case 3), we get, $-1 \leq \mathrm{A} \leq 0$. This shows that, $-1 \leq \mathrm{A} \leq 1$, and therefore, $-45^{\circ} \leq \theta_{\mathrm{FP}} \leq 45^{\circ}$.

2) For $\theta_{\mathrm{CP}}$ : In analogy to $\theta_{\mathrm{FP}}$, the expression of $\theta_{\mathrm{CP}}$ is given as,

$$
\theta_{\mathrm{CP}}=\tan ^{-1}(\mathrm{X})
$$

where,

$$
\mathrm{X}=\frac{m_{\mathrm{CP}} \mathrm{Span}(\mathrm{OC}-\mathrm{SC})}{\mathrm{OC} \times \mathrm{SC}+m_{\mathrm{CP}}^{2} \mathrm{Span}^{2}}
$$

The range of $\mathrm{X}$ can be obtained by considering two cases of the covariance matrices:

Case 1: $\mathbf{C}_{2(I)}=\frac{1}{4}\left[\begin{array}{cc}2 \rho+1 & i(2 \rho-1) \\ -i(2 \rho-1) & 2 \rho+1\end{array}\right] ; \quad 0 \leq \rho \leq 0.5$

The 2D Barakat degree of polarization, $m_{\mathrm{CP}}$ is expressed in terms of $\rho$ as,

$$
m_{\mathrm{CP}}=\left(1-\frac{8 \rho}{\left(4 \rho^{2}+4 \rho+1\right)}\right)^{\frac{1}{2}}
$$

and,

$$
\mathrm{X}=\frac{m_{\mathrm{CP}}\left(1-4 \rho^{2}\right)}{2 \rho+m_{C P}^{2}\left(4 \rho^{2}+4 \rho+1\right)}
$$

Using (31) and (32) in the range of $\rho$, we get $0 \leq \mathrm{X} \leq 1$.

For the second case,

Case 2: $\mathbf{C}_{2(I I)}=\frac{1}{4}\left[\begin{array}{cc}2 \rho+1 & -i(2 \rho-1) \\ i(2 \rho-1) & 2 \rho+1\end{array}\right] ; \quad 0.5 \leq \rho \leq 1$

with,

$$
m_{\mathrm{CP}}=\left(1-\frac{8 \rho}{\left(4 \rho^{2}+4 \rho+1\right)}\right)^{\frac{1}{2}}
$$

and,

$$
\mathrm{X}=\frac{m_{\mathrm{CP}}\left(4 \rho^{2}-1\right)}{2 \rho+m_{\mathrm{CP}}^{2}\left(4 \rho^{2}+4 \rho+1\right)}
$$

Using (34) and (35) in the range of $\rho$, we get $-1 \leq X \leq 0$. Therefore, combining the two ranges of $\mathrm{X}$ we get, $-1 \leq \mathrm{X} \leq$ 1 , and hence, $-45^{\circ} \leq \theta_{\mathrm{CP}} \leq 45^{\circ}$.

\section{B. Roll-invariant nature of $\theta_{\mathrm{FP}}$}

In order to show that $\theta_{\mathrm{FP}}$ is a roll-invariant parameter, let the coherency matrix $\mathbf{T}$ be unitarily rotated by $\mathbf{R}(\Psi)$ as,

$$
\mathbf{T}(\Psi)=\mathbf{R}(\Psi) \mathbf{T R}(\Psi)^{-1}
$$

where

$$
\mathbf{R}(\Psi)=\left[\begin{array}{ccc}
1 & 0 & 0 \\
0 & \cos 2 \Psi & \sin 2 \Psi \\
0 & -\sin 2 \Psi & \cos 2 \Psi
\end{array}\right]
$$

With this,

$$
\begin{gathered}
T_{11}(\Psi)=T_{11} \\
T_{22}(\Psi)=T_{22} \cos ^{2}(2 \Psi)+T_{32} \cos (2 \Psi) \sin (2 \Psi)+ \\
T_{23} \cos (2 \Psi) \sin (2 \Psi)+T_{33} \sin ^{2}(2 \Psi) \\
T_{33}(\Psi)=T_{22} \sin ^{2}(2 \Psi)-T_{32} \cos (2 \Psi) \sin (2 \Psi)- \\
T_{23} \cos (2 \Psi) \sin (2 \Psi)+T_{33} \cos ^{2}(2 \Psi)
\end{gathered}
$$


Therefore, $T_{11}(\Psi)-T_{22}(\Psi)-T_{33}(\Psi)=T_{11}-T_{22}-T_{33}$ and $T_{22}(\Psi)+T_{33}(\Psi)=T_{22}+T_{33}$ i.e., both $T_{11}-T_{22}-T_{33}$ and $T_{22}+T_{33}$ are independent of the unitary rotation by an angle $\Psi$. The total power (i.e., Span), and the 3D Barakat degree of polarization $m_{\mathrm{FP}}$ are independent of $\Psi$. Hence, we conclude that the proposed scattering-type parameter for FP SAR,

$$
\theta_{\mathrm{FP}}=\tan ^{-1}\left(\frac{m_{\mathrm{FP}} \operatorname{Span}\left(T_{11}-T_{22}-T_{33}\right)}{T_{11}\left(T_{22}+T_{33}\right)+m_{\mathrm{FP}}^{2} \operatorname{Span}^{2}}\right)
$$

is independent of $\Psi$, i.e., it is a roll-invariant parameter.

\section{Roll-invariant nature of $\theta_{\mathrm{CP}}$}

The $2 \times 2$ covariance matrix can be expressed in terms of the elements of the Stokes vector $\overrightarrow{\mathbf{S}}=\left[S_{0}, S_{1}, S_{2}, S_{3}\right]$ as

$$
\mathbf{C}_{2}=\frac{1}{2}\left[\begin{array}{cc}
S_{0}+S_{1} & S_{2}+i S_{3} \\
S_{2}-i S_{3} & S_{0}-S_{1}
\end{array}\right]
$$

Let the $\mathbf{C}_{2}$ matrix be unitarily rotated by $\mathbf{R}(\Psi)$ as $\mathbf{C}_{2}(\Psi)=$ $\mathbf{R}(\Psi) \mathbf{C}_{2} \mathbf{R}(\Psi)^{-1}$, where the rotation matrix is,

$$
\mathbf{R}(\Psi)=\left[\begin{array}{cc}
\cos (\Psi) & -\sin (\Psi) \\
\sin (\Psi) & \cos (\Psi)
\end{array}\right]
$$

The elements of the $\mathbf{C}_{2}(\Psi)$ matrix are:

$$
\begin{aligned}
c_{11}(\Psi)= & \cos ^{2} \Psi\left(S_{0}+S_{1}\right)-\cos \Psi \sin \Psi\left(S_{2}-i S_{3}\right) \\
& -\cos \Psi \sin \Psi\left(S_{2}+i S_{3}\right)+\sin ^{2} \Psi\left(S_{0}-S_{1}\right), \\
c_{12}(\Psi)= & \cos \Psi \sin \Psi\left(S_{0}+S_{1}\right)-\sin ^{2} \Psi\left(S_{2}-i S_{3}\right) \\
& +\cos ^{2} \Psi\left(S_{2}+i S_{3}\right)-\cos \Psi \sin \Psi\left(S_{0}-S_{1}\right), \\
c_{21}(\Psi)= & \cos \Psi \sin \Psi\left(S_{0}+S_{1}\right)+\cos ^{2} \Psi\left(S_{2}-i S_{3}\right) \\
& -\sin ^{2} \Psi\left(S_{2}+i S_{3}\right)-\cos \Psi \sin \Psi\left(S_{0}-S_{1}\right), \\
c_{22}(\Psi)= & \sin ^{2} \Psi\left(S_{0}+S_{1}\right)-\cos \Psi \sin \Psi\left(S_{2}-i S_{3}\right) \\
& -\cos \Psi \sin \Psi\left(S_{2}+i S_{3}\right)+\cos ^{2} \Psi\left(S_{0}-S_{1}\right) .
\end{aligned}
$$

The total power $S_{0}=c_{11}(\Psi)+c_{22}(\Psi)$ and the fourth element of the Stokes vector $S_{3}=-i\left(c_{12}(\Psi)-c_{21}(\Psi)\right)$ are independent of the rotation angle $\Psi$. Since $S_{0}$ and $S_{3}$ are independent of $\Psi$, then $\mathrm{SC}=\left(S_{0}-S_{3}\right) / 2$ and $\mathrm{OC}=$ $\left(S_{0}+S_{3}\right) / 2$ are also independent of $\Psi$, i.e. both parameters are roll-invariant.

Alongside, note that $\left|\mathbf{C}_{2}\right|$ and $\operatorname{Tr}\left(\mathbf{C}_{2}\right)$ are roll-invariant, where $|\cdot|$ is the determinant and $\operatorname{Tr}(\cdot)$ is the trace of the matrix. Therefore, the 2D Barakat degree of polarization, $m_{\mathrm{CP}}=\sqrt{1-\frac{4\left|\mathbf{C}_{2}\right|}{\left(\operatorname{Tr}\left(\mathbf{C}_{2}\right)\right)^{2}}}$ is also roll-invariant. Hence, we conclude that the proposed scattering-type parameter for $\mathrm{CP}$ SAR,

$$
\theta_{\mathrm{CP}}=\tan ^{-1}\left(\frac{m_{\mathrm{CP}} S_{0}(\mathrm{OC}-\mathrm{SC})}{\mathrm{OC} \times \mathrm{SC}+m_{\mathrm{CP}}^{2} S_{0}^{2}}\right)
$$

is independent of $\Psi$, i.e., it is a roll-invariant parameter.

\section{Relationships of $\theta_{\mathrm{FP}}$ with $\alpha$, and of $\theta_{\mathrm{CP}}$ with $\chi$}

1) $\theta_{\mathrm{FP}}$ with $\alpha$ : Note that

$$
\frac{T_{11}}{\text { Span }}=\sum_{i=1}^{3} p_{i} \cos ^{2} \alpha_{i},
$$

where $\left(\alpha_{i}\right)_{i=1,2,3}$ are the individual scattering-type parameters obtained from the $\alpha-\beta$ parametrization of the Cloude-Pottier target scattering vector.

Let,

$$
\tan \eta_{1}=\frac{T_{11}}{m_{\mathrm{FP}} \operatorname{Span}}=\frac{\sum_{i=1}^{3} p_{i} \cos ^{2} \alpha_{i}}{\sqrt{1-\prod_{i=1}^{3} 3 p_{i}}},
$$

and

$$
\tan \eta_{2}=\frac{T_{22}+T_{33}}{m_{\mathrm{FP}} \operatorname{Span}}=\frac{1-\sum_{i=1}^{3} p_{i} \cos ^{2} \alpha_{i}}{\sqrt{1-\prod_{i=1}^{3} 3 p_{i}}} .
$$

Then,

$$
\begin{aligned}
\tan \left(\eta_{1}-\eta_{2}\right) & =\frac{\frac{\sum_{i=1}^{3} p_{i} \cos ^{2} \alpha_{i}}{\sqrt{1-\prod_{i=1}^{3} 3 p_{i}}}-\frac{1-\sum_{i=1}^{3} p_{i} \cos ^{2} \alpha_{i}}{\sqrt{1-\prod_{i=1}^{3} 3 p_{i}}}}{1+\frac{\sum_{i=1}^{3} p_{i} \cos ^{2} \alpha_{i}}{\sqrt{1-\prod_{i=1}^{3} 3 p_{i}}} \frac{1-\sum_{i=1}^{3} p_{i} \cos ^{2} \alpha_{i}}{\sqrt{1-\prod_{i=1}^{3} 3 p_{i}}}} \\
& =\frac{\frac{2 \sum_{i=1}^{3} p_{i} \cos ^{2} \alpha_{i}-1}{\sqrt{1-\prod_{i=1}^{3} 3 p_{i}}}}{1+\frac{\left(\sum_{i=1}^{3} p_{i} \cos ^{2} \alpha_{i}\right)\left(1-\sum_{i=1}^{3} p_{i} \cos ^{2} \alpha_{i}\right)}{1-\prod_{i=1}^{3} 3 p_{i}}} .
\end{aligned}
$$

Hence, the expression of $\theta_{\mathrm{FP}}$ is given in terms of $\left(p_{i}\right)_{i=1,2,3}$ and $\left(\alpha_{i}\right)_{i=1,2,3}$ as,

$$
\begin{aligned}
\tan \theta_{\mathrm{FP}}= & \frac{\text { Num }}{\text { Den }}, \text { in which } \\
\text { Num }= & \left(2 \sum_{i=1}^{3} p_{i} \cos ^{2} \alpha_{i}-1\right) \sqrt{1-\prod_{i=1}^{3} 3 p_{i}, \text { and }} \\
\text { Den }= & 1-\prod_{i=1}^{3} 3 p_{i}+ \\
& \left(\sum_{i=1}^{3} p_{i} \cos ^{2} \alpha_{i}\right)\left(1-\sum_{i=1}^{3} p_{i} \cos ^{2} \alpha_{i}\right) .
\end{aligned}
$$

Therefore, it can be noticed that $\theta_{\mathrm{FP}}$ is a function of $\sum_{i=1}^{3} p_{i} \cos ^{2} \alpha_{i}$, and the 3D Barakat degree of polarization, $\sqrt{1-\prod_{i=1}^{3} 3 p_{i}}$. Similarly, in [43], $N_{11}=\sum_{i=1}^{3} p_{i} \cos ^{2} \alpha_{i}$ is defined as the surface scattering fraction and is interpreted as the fraction of odd-bounce scattering from total backscattered power. Furthermore, it can also be related to the fraction of right-left circularly polarized response from total backscattered power measured in a circular basis.

2) $\theta_{\mathrm{CP}}$ with $\chi$ : The ellipticity $\chi$ is defined in terms of the elements of Stokes vector, $\overrightarrow{\mathbf{S}}=\left[S_{0}, S_{1}, S_{2}, S_{3}\right]$ as

$$
\sin 2 \chi=-\frac{S_{3}}{m_{\mathrm{CP}} S_{0}},
$$

where $m_{\mathrm{CP}}$ is the $2 \mathrm{D}$ Barakat degree of polarization, and

$$
\text { OC }=\frac{S_{0}+S_{3}}{2} \quad \text { and } \quad \mathrm{SC}=\frac{S_{0}-S_{3}}{2} .
$$


Therefore, $\theta_{\mathrm{CP}}$ is given in terms of $\chi$ and $m_{\mathrm{CP}}$ as:

$$
\begin{aligned}
\tan \theta_{\mathrm{CP}} & =\frac{m_{\mathrm{CP}} \operatorname{Span}(\mathrm{OC}-\mathrm{SC})}{\mathrm{OC} \times \mathrm{SC}+m_{\mathrm{CP}}^{2} \mathrm{Span}^{2}} \\
& =\frac{m_{\mathrm{CP}} S_{0} S_{3}}{\frac{S_{0}^{2}-S_{3}^{2}}{4}+m_{\mathrm{CP}}^{2} S_{0}^{2}}=\frac{4 m_{\mathrm{CP}} S_{0} S_{3}}{S_{0}^{2}-S_{3}^{2}+4 m_{\mathrm{CP}}^{2} S_{0}^{2}} \\
& =\frac{4 m_{\mathrm{CP}}\left(S_{3} / S_{0}\right)}{1-\left(S_{3} / S_{0}\right)^{2}+4 m_{\mathrm{CP}}^{2}}=\frac{4 m_{\mathrm{CP}}\left(-m_{\mathrm{CP}} \sin 2 \chi\right)}{1-m_{\mathrm{CP}}^{2} \sin ^{2} 2 \chi+4 m_{\mathrm{CP}}^{2}} \\
& =-\frac{4 m_{\mathrm{CP}}^{2} \sin 2 \chi}{1-m_{\mathrm{CP}}^{2} \sin ^{2} 2 \chi+4 m_{\mathrm{CP}}^{2}} .
\end{aligned}
$$

\section{ACKNOWLEDGEMENT}

The authors would like to thank Prof. Dr. Eric Pottier, Prof. José J. Gil and Prof. Christian Brosseau for their valuable inputs to enrich the scientific content of this manuscript. The authors would like to thank MAXAR Technologies Ltd., formerly MacDonald, Dettwiler, and Associates (MDA), for providing RADARSAT-2 data and JAXA for providing the ALOS-2 PALSAR data. The authors acknowledge the GEOAWS Earth Observation Cloud Credits Program, through the project: "AWS4AgriSAR-Crop inventory mapping from SAR data on cloud computing platform". Mr. D. Ratha (co-author) would like to thank the Council of Scientific and Industrial Research (CSIR) for supporting his doctoral studies. Prof. A. C. Frery acknowledges support from CNPq and Fapeal (Brazi).

\section{REFERENCES}

[1] S. R. Cloude and E. Pottier, "An entropy based classification scheme for land applications of polarimetric SAR," IEEE Trans. Geosci. Remote Sens., vol. 35, no. 1, pp. 68-78, 1997.

[2] R. Touzi, "Target scattering decomposition in terms of roll-invariant target parameters," IEEE Trans. Geosci. Remote Sens., vol. 45, no. 1, pp. 73-84, 2006.

[3] D. Ratha, E. Pottier, A. Bhattacharya, and A. C. Frery, "A polsar scattering power factorization framework and novel roll-invariant parameterbased unsupervised classification scheme using a geodesic distance," IEEE Trans. Geosci. Remote Sens., pp. 1-17, 2019.

[4] A. Freeman and S. L. Durden, "A three-component scattering model for polarimetric SAR data," IEEE Trans. Geosci. Remote Sens., vol. 36, no. 3, pp. 963-973, 1998.

[5] J.-S. Lee, M. R. Grunes, T. L. Ainsworth, L.-J. Du, D. L. Schuler, and S. R. Cloude, "Unsupervised classification using polarimetric decomposition and the complex Wishart classifier," IEEE Trans. Geosci. Remote Sens., vol. 37, no. 5, pp. 2249-2258, 1999.

[6] J.-S. Lee, M. R. Grunes, E. Pottier, and L. Ferro-Famil, "Unsupervised terrain classification preserving polarimetric scattering characteristics," IEEE Trans. Geosci. Remote Sens., vol. 42, no. 4, pp. 722-731, 2004.

[7] Y. Yamaguchi, T. Moriyama, M. Ishido, and H. Yamada, "Fourcomponent scattering model for polarimetric SAR image decomposition," IEEE Trans. Geosci. Remote Sens., vol. 43, no. 8, pp. 1699-1706, 2005.

[8] M. Arii, J. J. van Zyl, and Y. Kim, "A general characterization for polarimetric scattering from vegetation canopies," IEEE Trans. Geosci. Remote Sens., vol. 48, no. 9, pp. 3349-3357, 2010.

[9] M. Neumann, L. Ferro-Famil, and A. Reigber, "Estimation of forest structure, ground, and canopy layer characteristics from multibaseline polarimetric interferometric SAR data," IEEE Trans. Geosci. Remote Sens., vol. 48, no. 3, pp. 1086-1104, 2009.

[10] J. J. Van Zyl, M. Arii, and Y. Kim, "Model-based decomposition of polarimetric SAR covariance matrices constrained for nonnegative eigenvalues," IEEE Trans. Geosci. Remote Sens., vol. 49, no. 9, pp. 3452-3459, 2011.

[11] Y. Cui, Y. Yamaguchi, J. Yang, H. Kobayashi, S.-E. Park, and G. Singh, "On complete model-based decomposition of polarimetric SAR coherency matrix data," IEEE Trans. Geosci. Remote Sens., vol. 52, no. 4, pp. 1991-2001, 2013.
[12] W. An, Y. Cui, and J. Yang, "Three-component model-based decomposition for polarimetric SAR data," IEEE Trans. Geosci. Remote Sens., vol. 48, no. 6, pp. 2732-2739, 2010.

[13] J.-S. Lee and T. L. Ainsworth, "The effect of orientation angle compensation on coherency matrix and polarimetric target decompositions," IEEE Trans. Geosci. Remote Sens., vol. 49, no. 1, pp. 53-64, 2010.

[14] Y. Yamaguchi, A. Sato, W.-M. Boerner, R. Sato, and H. Yamada, "Fourcomponent scattering power decomposition with rotation of coherency matrix," IEEE Trans. Geosci. Remote Sens., vol. 49, no. 6, pp. 22512258, 2011.

[15] G. Singh, Y. Yamaguchi, and S.-E. Park, "General four-component scattering power decomposition with unitary transformation of coherency matrix," IEEE Trans. Geosci. Remote Sens., vol. 51, no. 5, pp. 30143022, May 2013.

[16] A. Bhattacharya, G. Singh, S. Manickam, and Y. Yamaguchi, "An adaptive general four-component scattering power decomposition with unitary transformation of coherency matrix (AG4U)," IEEE Geosci. Remote Sens. Lett., vol. 12, no. 10, pp. 2110-2114, 2015.

[17] S.-W. Chen, X.-S. Wang, S.-P. Xiao, and M. Sato, "General polarimetric model-based decomposition for coherency matrix," IEEE Trans. Geosci. Remote Sens., vol. 52, no. 3, pp. 1843-1855, 2013.

[18] A. Bhattacharya, A. Muhuri, S. De, S. Manickam, and A. C. Frery, "Modifying the Yamaguchi four-component decomposition scattering powers using a stochastic distance," IEEE J. Sel. Topics Appl. Earth Observ. Remote Sens., vol. 8, no. 7, pp. 3497-3506, July 2015.

[19] T. Eltoft and A. P. Doulgeris, "Model-based polarimetric decomposition with higher order statistics," IEEE Geosci. Remote Sens. Lett., vol. 16, no. 6, pp. 992-996, 2019.

[20] W. An and M. Lin, "A reflection symmetry approximation of multilook polarimetric SAR data and its application to Freeman-Durden decomposition," IEEE Trans. Geosci. Remote Sens., vol. 57, no. 6, pp. 3649-3660, 2019.

[21] Z. Jiao, J. Yang, C. Yeh, and J. Song, "Modified three-component decomposition method for polarimetric sar data," IEEE Geosci. Remote Sens. Lett., vol. 11, no. 1, pp. 200-204, Jan 2014.

[22] Z. Shuang, Y. Xiangchuan, and W. Lu, "Modified version of threecomponent model-based decomposition for polarimetric SAR data," $J$. Syst. Eng. Electron., vol. 30, no. 2, pp. 270-277, 2019.

[23] S.-W. Chen, Y.-Z. Li, X.-S. Wang, S.-P. Xiao, and M. Sato, "Modeling and interpretation of scattering mechanisms in polarimetric synthetic aperture radar: Advances and perspectives," IEEE Signal Process. Mag., vol. 31, no. 4, pp. 79-89, 2014.

[24] J.-C. Souyris, P. Imbo, R. Fjortoft, S. Mingot, and J.-S. Lee, "Compact polarimetry based on symmetry properties of geophysical media: the $\pi / 4$ mode," IEEE Trans. Geosci. Remote Sens., vol. 43, no. 3, pp. 634 646, 2005 .

[25] N. Stacy and M. Preiss, "Compact polarimetric analysis of X-band SAR data," EUSAR 2006, 2006.

[26] R. K. Raney, "Hybrid-polarity SAR architecture," IEEE Trans. Geosci. Remote Sens., vol. 45, no. 11, pp. 3397-3404, 2007.

[27] G. G. Stokes, "On the composition and resolution of streams of polarized light from different sources," Transactions of the Cambridge Philosophical Society, vol. 9, p. 399, 1851.

[28] R. K. Raney, J. T. S. Cahill, G. W. Patterson, and D. B. J. Bussey, "The m-chi decomposition of hybrid dual-polarimetric radar data with application to lunar craters," Journal of Geophysical Research: Planets, vol. 117, no. E12, pp. n/a-n/a, 2012. [Online]. Available: http://dx.doi.org/10.1029/2011JE003986

[29] A. Bhattacharya, S. De, A. Muhuri, M. Surendar, G. Venkataraman, and A. Das, "A new compact polarimetric SAR decomposition technique," Remote Sensing Letters, vol. 6, no. 12, pp. 914-923, 2015.

[30] S. R. Cloude and E. Pottier, "A review of target decomposition theorems in radar polarimetry," IEEE Trans. Geosci. Remote Sens., vol. 34, no. 2, pp. 498-518, 1996.

[31] A. Swartz, H. Yueh, J. Kong, L. Novak, and R. Shin, "Optimal polarizations for achieving maximum contrast in radar images," Journal of Geophysical Research: Solid Earth, vol. 93, no. B12, pp. 15252 $15260,1988$.

[32] R. Barakat, "Degree of polarization and the principal idempotents of the coherency matrix," Opt. Commun., vol. 23, no. 2, pp. 147-150, 1977.

[33] — , "n-fold polarization measures and associated thermodynamic entropy of n partially coherent pencils of radiation," Optica Acta: International Journal of Optics, vol. 30, no. 8, pp. 1171-1182, 1983.

[34] P. Réfrégier, F. Goudail, P. Chavel, and A. Friberg, "Entropy of partially polarized light and application to statistical processing techniques," JOSA A, vol. 21, no. 11, pp. 2124-2134, 2004. 
[35] S. Cloude and E. Pottier, "An entropy based classification scheme for land applications of polarimetric SAR," IEEE Trans. Geosci. Remote Sens., vol. 35, no. 1, pp. 68-78, 1997.

[36] D. J. McLaughlin, N. Allan, E. M. Twarog, and D. Trizna, "High resolution polarimetric radar scattering measurements of low grazing angle sea clutter," IEEE J. Ocean. Eng., vol. 20, no. 3, pp. 166-178, 1995.

[37] I. Brown, S. Mwansasu, and L.-O. Westerberg, "L-band polarimetric target decomposition of mangroves of the Rufiji Delta, Tanzania," Remote Sensing, vol. 8, no. 2, p. 140, 2016.

[38] S.-H. Hong and S. Wdowinski, "Double-bounce component in crosspolarimetric SAR from a new scattering target decomposition," IEEE Trans. Geosci. Remote Sens., vol. 52, no. 6, pp. 3039-3051, 2013.

[39] T. Ainsworth, D. Schuler, and J.-S. Lee, "Polarimetric SAR characterization of man-made structures in urban areas using normalized circularpol correlation coefficients," Remote Sens. Environ., vol. 112, no. 6, pp. 2876-2885, 2008.

[40] R. Sabry and P. Vachon, "A unified framework for general compact and quad polarimetric SAR data and imagery analysis," IEEE Trans. Geosci. Remote Sens., vol. 52, no. 1, pp. 582-602, Jan 2014.

[41] R. Guinvarch and L. Thirion-Lefevre, "Cross-polarization amplitudes of obliquely orientated buildings with application to urban areas," IEEE Geosci. Remote Sens. Lett., vol. 14, no. 11, pp. 1913-1917, 2017.

[42] D. K. Atwood and L. Thirion-Lefevre, "Polarimetric phase and implications for urban classification," IEEE Trans. Geosci. Remote Sens. vol. 56, no. 3, pp. 1278-1289, 2017.

[43] J. Praks, E. C. Koeniguer, and M. T. Hallikainen, "Alternatives to target entropy and alpha angle in sar polarimetry," IEEE Trans. Geosci. Remote Sens., vol. 47, no. 7, pp. 2262-2274, 2009.

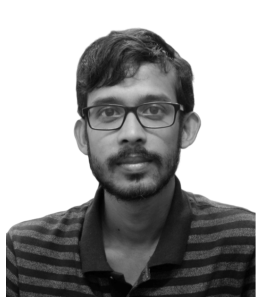

Subhadip Dey (S'17) received the B.Tech degree in Agricultural Engineering from Bidhan Chandra Krishi Viswavidyalaya, India. He received the M. Tech degree in Aquacultural Engineering, Department of Agricultural and Food Engineering, Indian Institute of Technology Kharagpur, India. He is currently pursuing the Ph.D. degree at the Microwave Remote Sensing Lab, Centre of Studies in Resources Engineering (CSRE), Indian Institute of Technology Bombay, India. His current research interests are land cover classification and agricultural crop mapping and monitoring using Synthetic Aperture Radar data.

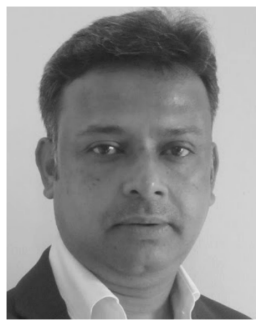

Avik Bhattacharya (M'08-SM'16) received the integrated M.Sc. degree in Mathematics from the Indian Institute of Technology, Kharagpur, India, in 2000 and the Ph.D. degree in remote sensing image processing and analysis from Télécom ParisTech, Paris, France, and the Ariana Research Group, Institut National de Recherche en Informatique et en Automatique (INRIA), Sophia Antipolis, Nice, France, in 2007. He is currently an Associate Professor at the Centre of Studies in Resources Engineering, Indian Institute of Technology Bombay (CSRE, IITB), Mumbai, India. Before joining IITB, he was a Canadian Government Research Fellow at the Canadian Centre for Remote Sensing (CCRS) in Ottawa, ON, Canada. He received the Natural Sciences and Engineering Research Council of Canada visiting scientist fellowship at the Canadian national laboratories, from 2008 to 2011. His current research interests include SAR polarimetry, statistical analysis of polarimetric SAR images, applications of Radar Remote Sensing in Agriculture, Cryosphere, Urban and Planetary studies. Dr. Bhattacharya is the Editor-in-Chief of IEEE GEOSCIENCE AND REMOTE SENSING LETTERS (GRSL). He was an Associate Editor of IEEE GRSL. He has been the Guest Editor of the special issue on Applied Earth Observations and Remote Sensing in India in IEEE JOURNAL OF SELECTED TOPICS IN APPLIED EARTH OBSERVATIONS AND REMOTE SENSING (J-STARS), 2017. He was one of the guest editors of the special stream on Advanced Statistical Techniques in SAR Image Processing and Analysis in IEEE GEOSCIENCE AND REMOTE SENSING LETTERS, 2018. He is the Founding Chairperson of the IEEE Geoscience and Remote Sensing Society (GRSS) Chapter of the Bombay Section. He is currently leading the Microwave Remote Sensing Lab (www.mrslab.in) at CSRE, IITB

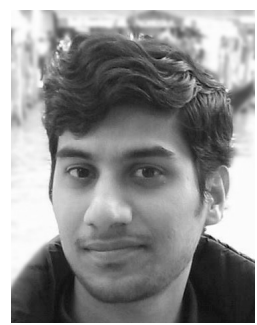

Debanshu Ratha (S'16) received his 5 years Integrated M.Sc in Mathematics from NISER Bhubaneswar, in 2013. Since then, he has worked in full-time research positions at the DIAM, TU Delft, The Netherlands, and the CSRE, IIT Bombay, India. Currently, he is pursuing his Ph.D. degree as a CSIR fellowship awardee at CSRE, IIT Bombay. His research interests include Theory of Remote Sensing and its Applications, Radar Polarimetry, Variational Analysis, Information Geometry, Graph Theory, Machine Learning, Data Fusion. He is a recipient of many prestigious scholarships and visiting fellowships in the past. Recently, he has been a Visiting Researcher at the IETR Laboratory, University of Rennes 1, under the Raman Charpak Fellowship Programme.

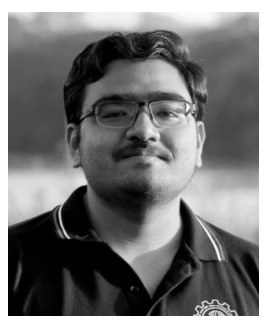

Dipankar Mandal (S'16) received the B.Tech. degree in agricultural engineering from Bidhan Chandra Krishi Viswavidyalaya, India, in 2015. He is currently working towards the M.Tech + Ph.D. dual degree in geoinformatics and natural resources engineering in the Centre of Studies in Resources Engineering, Indian Institute of Technology Bombay, Mumbai, India. Mr. Mandal was the recipient of the Shastri Research Student Fellowship 2018-19 Award by the Shastri Indo-Canadian Institute, India and was a visiting researcher in Agriculture and Agri-Food Canada (AAFC) and Carleton University, Ottawa, Canada from October 2018 to February 2019. As a visiting researcher, he contributed to SAR Intercomparison experiment for crop biophysical parameter estimation within the Joint Experiment for Crop Assessment and Monitoring (JECAM) network of GEO Global Agricultural Monitoring. His research interest includes applications of SAR polarimetry for crop classification, vegetation biophysical parameter estimation, deriving vegetation indices, and yield forecasting.

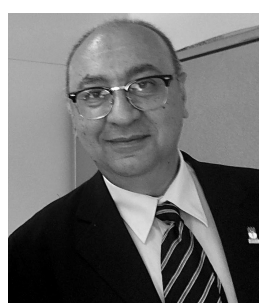

Alejandro C. Frery (S'92-SM'03) received the B.Sc. degree in Electronic and Electrical Engineering from the Universidad de Mendoza, Mendoza, Argentina. His M.Sc. degree was in Applied Mathematics (Statistics) from the Instituto de Matemática Pura e Aplicada (IMPA, Rio de Janeiro) and his Ph.D. degree was in Applied Computing from the Instituto Nacional de Pesquisas Espaciais (INPE, São José dos Campos, Brazil). He is currently the leader of LaCCAN - Laboratório de Computação Científica e Análise Numérica, Universidade Federal de Alagoas, Maceió, Brazil, and holds a Huashan Scholar position (20192021) with the Key Lab of Intelligent Perception and Image Understanding of the Ministry of Education, Xidian University, Xi'an, China. His research interests are statistical computing and stochastic modeling. 